Cahiers Charlevoix

Études franco-ontariennes
Cahiers Charlevoix Études franco-ontariennes

or Crevereerie of:

\title{
Passage de l'élémentaire au secondaire et décrochage culturel en Ontario français. Élimination de quelques facteurs
}

\section{Simon Laflamme}

Volume 9, 2012

URI : https://id.erudit.org/iderudit/1039312ar

DOI : https://doi.org/10.7202/1039312ar

Aller au sommaire du numéro

\section{Éditeur(s)}

Société Charlevoix

Presses de l’Université d’Ottawa

ISSN

1203-4371 (imprimé)

2371-6878 (numérique)

Découvrir la revue

Citer cet article

Laflamme, S. (2012). Passage de l'élémentaire au secondaire et décrochage culturel en Ontario français. Élimination de quelques facteurs. Cahiers

Charlevoix, 9, 15-59. https://doi.org/10.7202/1039312ar

\section{Résumé de l'article}

Simon Laflamme examine le rôle qu'a pu jouer en Ontario français une " idéologie pro-anglaise » qui expliquerait le décrochage d'un certain nombre d'élèves du système scolaire français en faveur du système anglais. Mais avant d'étudier les tenants et aboutissants de cette idéologie, l'auteur entend vérifier si le comportement des jeunes, tant francophones qu'anglophones, devant la transition du cycle élémentaire au cycle secondaire, est comparable. Et il le fait à partir de données recueillies auprès de jeunes, de parents et d'éducateurs entre les mois d'octobre et de décembre 2008, dans le cadre d'une étude menée en Ontario prenant en compte toute une série de facteurs associés à ce passage : profil des deux échantillons (selon le groupe d'appartenance, le pays de naissance, la discrimination, la langue parlée et le statut socio-économique de la famille d'origine), l'influence des amis, l'estime de soi, la manière dont on se sent à l'école, l'évaluation de l'école et l'impression sur la diversité de l'école, l'opinion sur les enseignants, l'encouragement des parents et des enseignants, l'importance de l'école secondaire dans l'environnement de l'élève, l'aide pour le travail scolaire, le bonheur à l'école, les notes, le choix de l'école secondaire et les sentiments attachés à la transition. Au terme de la comparaison de tous ces facteurs, Simon Laflamme constate que les élèves franco-ontariens ne paraissent en rien différer de leurs semblables anglo-ontariens au moment de franchir le seuil de l'école secondaire. Ce qui lui fait « conclure que la dualité idéologique trouve son origine ailleurs » et qui devrait le conduire à une nouvelle " enquête qui voudrait comprendre le développement de l'idéologie duelle franco-ontarienne ", en considérant les artisans et les sources de ces discours.
Ce document est protégé par la loi sur le droit d'auteur. L'utilisation des services d’Érudit (y compris la reproduction) est assujettie à sa politique d'utilisation que vous pouvez consulter en ligne.

https://apropos.erudit.org/fr/usagers/politique-dutilisation/ 


\title{
Passage de l'élémentaire au secondaire et décrochage culturel en Ontario français. Élimination de quelques facteurs
}

\author{
Simon LAFLAMme \\ Département de sociologie \\ Université Laurentienne
}




\section{SoMmaIRE}

1. INTRODUCTION 17

2. Méthodologie 24

3. LES ÉLÈVES FRANCOPHONES ET LES ÉLÈVES ANGLOPHONES DEVANT LA TRANSITION 25

3.1. Profil des deux échantillons 25

3.1.1. Le groupe auquel on appartient 25

3.1.2. Le pays de naissance $\quad 27$

3.1.3. La discrimination 28

3.1.4. La langue 29

3.1.5. Le statut socio-économique de la famille d'origine $\quad 30$

3.2. Les amis 32

3.3. Soi et les autres 33

3.4. Manière dont on se sent à l'école 35

3.5. Évaluation de l'école $\quad 36$

3.6. Impression sur la diversité de l'école 37

3.7. Opinion sur les enseignants 38

3.8. Encouragement des parents et des enseignants $\quad 39$

3.9. Importance de l'école secondaire dans l'environnement de l'élève 41

3.10. Aide pour le travail scolaire 41

3.11. Est-on heureux à l'école? 43

3.12. Les notes 46

3.13. Choix de l'école secondaire 46

3.14. Sentiments attachés à la transition 51

4. Conclusion 55 


\section{Passage de l'élémentaire au secondaire et décrochage culturel en Ontario français. Élimination de quelques facteurs ${ }^{1}$}

Il existe en Ontario français une idéologie pro-anglaise à laquelle s'alimentent bon nombre de jeunes qui passent du système scolaire français au système anglais. Des parents de ces jeunes s'en nourrissent également. Éventuellement, des recherches devront porter sur les déterminants de cette idéologie et sur les façons dont elle circule. Mais avant d'examiner ce phénomène, il nous semble nécessaire de nous demander si le vécu familial et scolaire des élèves francophones et anglophones est comparable, si la manière dont les uns entrevoient la transition de l'élémentaire au secondaire est semblable à celle des autres. Tel est le propos de cette étude.

\section{INTRODUCTION}

Émile Durkheim (1858-1917) est considéré comme le père de la sociologie française, comme l'un des principaux fondateurs de la sociologie. Or il vient à la sociologie avec une réflexion largement orientée vers l'éducation : à la Faculté des lettres de Bordeaux, il est chargé d'un cours de " sciences sociales et pédagogie »; la chaire qu'il occupera à la Sorbonne à compter de 1902 est d'abord celle de " science de l'éducation », avant de devenir celle de « science de l'éducation et sociologie ». Comment donc Durkheim comprend-il son objet? À maints égards, l'éducation,

1. Cette étude n'aurait pas été possible sans la collaboration de plusieurs personnes que nous nous empressons de remercier : Bruce Ferguson et Kate Tilleczek, qui m'ont invité à intégrer leur équipe de recherche et avec qui j'ai eu des échanges dont cette étude a bénéficié ; Mélanie Girard, qui m’a assisté dans les analyses et qui a participé à la collecte des données ; Dara Roth Edney, qui a géré le projet et qui a participé à la collecte de données ; Dana Cudney et Siobhan Cardoso, qui ont contribué à la collecte des données ; Demetra Saldaris et Megan Börner qui ont animé plusieurs conversations. 
pour lui, c'est l'école, dont fait partie l'université. L'école est, à ses yeux, un agent fondamental de socialisation et de formation. Il estime que, avec la modernité, la société se densifie et croît, que le travail se divise. Il devient ainsi de moins en moins possible pour la famille de former les enfants en vue du travail et de transmettre les valeurs et les normes qui assurent une certaine homogénéité à l'ensemble social. La modernité fait en sorte que les sociétés ne peuvent se reproduire que dans la mesure où des écoles préparent les individus à une multitude d'emplois et leur enseignent les modes à partir desquels ils peuvent coexister. Avec la modernité, une collectivité ne peut se reproduire que si elle porte en elle un système d'éducation'. Tel est l'enseignement de Durkheim. À ses origines, donc, par une de ses manifestations, la sociologie est le constat de la nécessité du lien entre collectivité moderne et système d'éducation.

Avec le temps, cette corrélation essentielle n'a que gagné en évidence. Des États, de plus en plus nombreux, ont rendu l'école obligatoire. Au Canada, l'instruction s'est massifiée. Qu'on en prenne pour illustration les seules études postsecondaires :

L'ensemble des effectifs du niveau postsecondaire a plus que sextuplé entre 1951 et 1975, passant de 91100 à 592 100. Le taux de scolarisation est passé de $6 \%$ de la population âgée de 18 à 24 ans à $20 \%$. Au cours de la même période, les effectifs non universitaires ont augmenté de huit fois, passant de 27600 à 221000 , et les effectifs universitaires ont presque sextuplé, passant de 63500 à $371100^{3}$.

2. Émile Durkheim, Éducation et sociologie, Paris, Puf, 1922, version électronique dans «Les classiques des sciences sociales » : http://classiques.uqac.ca/classiques/Durkheim_emile/education_socio/education_socio.pdf ; L'Éducation morale, Paris, Puf, 1902-1903, version électronique dans « Les classiques des sciences sociales » : http://classiques.uqac.ca/classiques/Durkheim_emile/education_morale/education_morale.pdf; L'Évolution pédagogique en France (1904-1905), Paris, PuF, 1938, version électronique dans «Les classiques des sciences sociales »: http://classiques. uqac.ca/classiques/Durkheim_emile/evolution_ped_france/evol_ped_france_1.pdf.

3. M. Wisenthal, Statistiques historiques du Canada, Section W : éducation, Enseignement post secondaire (séries W307-532), Ottawa, Statistique Canada, http:// www.statcan.gc.ca/pub/11-516-x/sectionw/4147445-fra.htm, site consulté en mars 2009. 
En 2005-2006, les effectifs des collèges étaient de 613 503, ceux des universités, de $1047507^{4}$.

Le principe vaut pour les États. Il vaut aussi pour les populations minoritaires. Il vaut très certainement pour la minorité francophone en Ontario. C'est la raison pour laquelle ses leaders ont depuis longtemps revendiqué le droit à une scolarité en français et veillent continûment à ce que cette instruction soit de qualité ; c'est aussi pourquoi ils ont milité pour la création de conseils scolaires homogènes dans leur langue et de collèges unilingues français ; c'est pourquoi plusieurs militent encore pour l'avènement d'une université de langue française, qui remplacerait les institutions bilingues. Aux personnes qui résistent à la création d'institutions postsecondaires homogènes de langue française, des Gaétan Gervais rappellent des vérités semblables à celles que communiquait Durkheim :

L'université et le collège sont appelés à remplir deux grands rôles dans les sociétés minoritaires : la formation des élites (les chefs de file, les gens éduqués, des experts dans tous les domaines) et l'épanouissement de la culture (en devenant un support social culturel). En fait ces institutions sont des éléments essentiels pour la continuité et pour la reproduction d'une sociétés.

Bon nombre de chercheurs du Canada français examinent les minorités en réaffirmant l'inhérence de la culture collective à la scolarisation. Selon eux, la vitalité des communautés de langue française dépend du rôle que jouent en elles les institutions - dans leur acception sociologique - et, au premier chef, l'école. Plusieurs travaux qui sont effectués autour de Rodrigue Landry en

4. Statistique Canada, Tableaux par sujet : éducation, formation et apprentissage, http://www40.statcan.ca/102/ind01/13_1821-fra.htm?hili_none.

5. Gaétan Gervais, " Pour de meilleures structures institutionnelles au niveau universitaire », dans Clinton Archibald (dir.), Actes du colloque national sur l'enseignement postsecondaire en langue française à l'extérieur du Québec. Situation actuelle et recherche de scénarios d'un développement d'aide aux communautés francophones hors Québec (Ottawa, les 10, 11 et 12 mai 1985), Ottawa, Fédération des francophones hors Québec, 1985, p. 31. 
témoignent ${ }^{6}$. Puisque l'instruction se révèle comme déterminante du sort d'une collectivité, les recherches se consacrent souvent à en mesurer les tenants et aboutissants. Un ouvrage qui fait état d'un net désavantage pour une communauté mobilise alors ses leaders et invite ses chercheurs à pousser les analyses. C'est bien l'effet qu'a obtenu la publication du rapport de Stacy Churchill, Saeed Quazi et Normand Frenette qui dévoilait en 1985 que la population franco-ontarienne était l'une des moins instruites du Canada et que la probabilité pour ses individus de faire des études postsecondaires était nettement inférieure à celle de l'ensemble des autres Ontariens ${ }^{7}$. Ces révélations ont servi de justification à plusieurs revendications et suscité un grand nombre de recherches sur le rôle de l'école de même que sur les causes et les effets des aspirations éducationnelles ${ }^{8}$.

6. Rodrigue Landry, Vers une politique scolaire des langues, Fredericton, Ministère de l'éducation, 1985 ; Rodrigue Landry, Réal Allard et Kenneth Deveau, Profil sociolangagier des élèves de $11^{e}$ année des écoles de langue française de l'Ontario. Outil de réflexion sur les défis de l'aménagement linguistique en éducation, Moncton, Institut canadien de recherche sur les minorités linguistiques, 2007 ; Rodrigue Landry, Réal Allard et Denis Haché, Développement psycholangagier d'élèves francoontariens à la fin du cycle secondaire, Moncton/Sudbury, Centre de recherche et de développement en éducation / Centre de recherche en éducation du Nouvel-Ontario, 1995 ; Rodrigue Landry, Sophie Le Touzé, Anne Gilbert et Joseph-Yvon Thériault, Le Point de vue des enseignantes et des enseignants sur les défis de l'enseignement en milieu minoritaire, Rapport d'enquête, Ottawa, Centre interdisciplinaire de recherche sur la citoyenneté et les minorités (CIRCEM), Institut canadien de recherche sur les minorités linguistiques / Fédération canadienne des enseignantes et des enseignants, 2004 ; Rodrigue Landry et Serge Rousselle, Éducation et droits collectifs : au-delà de l'article 23 de la Charte, Moncton, Les Éditions de la Francophonie, 2003.

7. Stacy Churchill, Saeed Quazi et Normand Frenette, Education et besoins des Franco-Ontariens : le diagnostic d'un système d'éducation, vol. I : Problèmes de l'ensemble du système : l'élémentaire et le secondaire, Toronto, Conseil de l'éducation franco-ontarienne, 1985 ; Stacy Churchill, Saeed Quazi et Normand Frenette, Éducation et besoins des Franco-Ontariens : le diagnostic d'un système d'éducation, vol. II : Le postsecondaire, Toronto, Conseil de l'éducation franco-ontarienne, 1985.

8. Par exemple : Jean-Charles Cachon, Étude stratégique sur les services d'éducation universitaire en français dans le Nord-Est de l'Ontario, Sudbury, Université Laurentienne, 1986 ; Simon Laflamme et Donald Dennie, L'Ambition démesurée. Enquête sur les aspirations et les représentations des étudiants et des étudiantes francophones du Nord-Est de l'Ontario, Sudbury, Institut franco-ontarien / Éditions Prise de parole, «Collection Universitaire : série Études », 1990 ; Pierre Poirier, Évelyn Gagné et Maurice Lapointe, Le Profil de l'étudiant franco-ontarien quant à son intentionnalité de poursuivre des études post-secondaires, Ottawa, Faculté d'éducation, Université d'Ottawa, 1987. 
Il est important pour une communauté minoritaire de disposer d'établissements d'enseignement, de la maternelle au postsecondaire. Mais il est nécessaire que ses enfants fréquentent les écoles qui, théoriquement, sont conçues pour les accueillir. Or, certaines études ont mis en évidence une diminution des effectifs au long du parcours scolaire. Cindy-Lynne Tremblay en a fait la démonstration dans une communication des Journées de l'AcFAsSudbury ${ }^{9}$. Une autre preuve en a été donnée dans un article de Francophonies d'Amérique ${ }^{10}$. Sur le site de Patrimoine canadien, on peut lire ce qui suit :

Le pourcentage des élèves de l'Ontario fréquentant les écoles de langue française en 2003-2004 nous indique une perte de 31000 élèves sur un continuum de la maternelle à la $12^{\text {ième }}$ année. Cette perte est plus marquée entre la maternelle et la $1^{\text {ère }}$ année, entre la $6^{\text {ième }}$ et la $7^{\text {ième }}$ année et entre la $8^{\text {ième }}$ et la 9 ième année. Les projections EQM de 2005-2006 donnent un nombre de 81794 élèves sur un potentiel d'effectifs de 110456 élèves, donc un écart de 28662 élèves (12 361 élèves à l'élémentaire et 16301 élèves au secondaire). Selon les données provisoires fournies par les conseils scolaires dans le cadre du Programme axé sur la réussite des élèves, le taux annuel

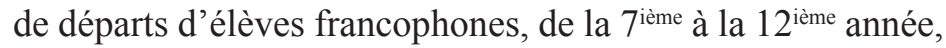
vers le système anglophone en Ontario en 2004-2005 est de $4,3 \%$. D'autre part, d'après les renseignements fournis par les conseils scolaires et les écoles, le taux d'obtention du diplôme après cinq années d'études secondaires en 2004-2005, est de $72 \%$; donc un taux d'abandon scolaire provincial qui pourrait s'établir autour de $28 \%{ }^{11}$.

9. Cindy-Lynne Tremblay, " "Décrochage culturel" au secondaire en Ontario français », dans Julie Boissonneault et Marie Bernier (dir.), Actes de la $13^{e}$ journée Sciences et savoirs, Sudbury, AcFAs-Sudbury, 2007, p. 79-89.

10. Jacques Michaud, Julie Boissonneault, Daniel Côté, Cindy-Lynne Tremblay et Gratien Allaire, « Décrochage, raccrochage et rétention scolaires selon les jeunes franco-ontariens ", Francophonies d'Amérique, n 25, 2008, p. 125-155.

11. Plans d'affaires 2005-2006 à 2008-2009 Canada-Ontario, 2005-2006 à 20082009 relatifs aux fonds complémentaires en appui à l'amélioration de l'éducation en langue française, Patrimoine canadien, http://www.canadianheritage.gc.ca/pgm/lo-ol/ entente-agreement/education/ont/2005-2007/business-plan-fra.cfm (site consulté en mars 2010), février 2006. 
Le phénomène du décrochage scolaire des jeunes franco-ontariens a attiré l'attention de certains chercheurs. Ces chercheurs découvrent des causes de risque qui correspondent largement à celles qu'on observe dans les travaux qui portent sur les autres populations : les antécédents de l'élève, soit des malaises de nature familiale, scolaire ou communautaire ; des facteurs non liés à l'école, comme la réalité familiale et sociale, l'identité, l'expérience de vie ; des facteurs scolaires, comme la participation aux activités scolaires, le programme, le manque de soutien ${ }^{12}$. Le cas du décrochage culturel a été peu étudié et les spécialistes du décrochage chez les jeunes francophones de l'Ontario réclament pertinemment une recherche approfondie.

Le ministère de l'Éducation de l'Ontario vient de publier une étude qui a abordé la question ${ }^{13}$. Cette recherche porte sur la transition de l'école élémentaire à l'école secondaire. La question du transfert linguistique a été clairement posée aux jeunes qui ont participé à l'enquête et qui étudiaient dans les écoles françaises.

12. GratienAllaire,JacquesMichaud,JulieBoissonneault,DanielCôté,Pounthioun Diallo, Le Décrochage au secondaire en Ontario français, rapport soumis par l'Institut franco-ontarien à la Direction des politiques et programmes d'éducation en langue française, Ministère de l'Éducation de l'Ontario, Sudbury, Institut francoontarien, octobre 2005 ; Julie Boissonneault, Jacques Michaud, Daniel Côté, CindyLynne Tremblay et Gratien Allaire, "L'Abandon scolaire en Ontario français et perspectives d'avenir des jeunes ", Éducation et francophonie, vol. 35, no 1, printemps 2007, p. 3-22 ; Daniel Côté, Julie Boissonneault, Jacques Michaud, CindyLynne Tremblay et Gratien Allaire, « Dropping out of School : recommendations of Ontario Francophone Youths on how to Deal with the Problem », International Journal of Environmental, Cultural, Economic and Social Sustainability, vol. 3, n ${ }^{\circ} 4$, 2007, p. 153-161 ; Daniel Côté, Julie Boissonneault, Jacques Michaud, Cindy-Lynne Tremblay et Gratien Allaire, « On Disengaging from High School : Francophone Youths, Parents, and Educators in Ontario Speak out », dans Kate Tilleczek (dir.), Why Do Students Drop out of High School? Narrative Studies and Social Critiques, Lewiston (NY), Edwin Mellen Press, 2008, p. 115-142 ; Jacques Michaud, Julie Boissonneault, Daniel Côté, Cindy-Lynne Tremblay et Gratien Allaire, « Décrochage, raccrochage et rétention scolaires selon les jeunes franco-ontariens », op. cit.

13. Kate Tilleczek, Simon Laflamme, Bruce Ferguson, Dara Roth Edney, Mélanie Girard, Dana Cudney, Siobhan Cardoso, Nouveaux départs, faux départs : jeunes en transition de l'école élémentaire à l'école secondaire (traduction de Fresh Starts and False Starts : A Final Report of the Transition Study. Mapping the Processes and Pathways of Transition from Elementary to Secondary School in Ontario), pour la Direction de la mise en œuvre, de la formation et de l'évaluation dans le cadre des initiatives de la réussite des élèves et de l'apprentissage jusqu'à l'âge de 18 ans du ministère de l'Éducation de l'Ontario, Toronto, 2010. 
L'analyse des réponses qui ont été fournies permet de constater :

i. que, aux yeux de bon nombre de jeunes francophones, étudier en français est synonyme d'être bilingue et que le bilinguisme est promesse d'emploi valorisant ;

ii. que, pour certains jeunes francophones, le fait d'étudier en français assure un bilinguisme qui, lui, étend les possibilités de la communication avec les autres ;

iii. que, pour plusieurs jeunes francophones, un transfert linguistique est difficilement concevable ;

iv. que les discours pro-anglais et anti-français sont actifs ;

v. que le passage à l'anglais se nourrit très aisément des discours anti-français et pro-anglais et qu'il le fait d'autant plus facilement que les aléas de la vie le rendent possible ;

vi. que, selon certains jeunes, il ne sert à rien de chercher à convaincre d'étudier en français les élèves qui n'aiment pas le français ${ }^{14}$.

Ces observations sont éclairantes. Elles font état d'un discours dont certains des propos sont antinomiques, et cette contradiction est peu contestable puisqu'elle rend compte aussi bien de l'inclination pour les études en français que du transfert vers les écoles de langue anglaise.

Il faudra, certes, consolider ces résultats par d'autres recherches, comprendre comment se conjuguent des opinions paradoxales, comment elles circulent dans la communauté francophone, et selon quelles influences. Mais on doit au préalable se demander si les élèves francophones et anglophones se distinguent par d'autres aspects ou s'ils ne diffèrent que sur les représentations linguistiques. Il importe, en effet, de vérifier s'ils perçoivent leur milieu de façon semblable, si leurs expériences scolaires sont similaires, s'ils envisagent pareillement la transition de l'élémentaire au secondaire quand on fait abstraction des représentations linguistiques, sans pour autant négliger les réalités communicationnelles. Car, si tel n'est pas le cas, c'est 
par référence à la dissimilitude qu'on devrait expliquer le fait que dans la population des jeunes francophones on observe un discours duel et que plusieurs élèves saisissent l'occasion que leur offre la transition pour changer de langue de scolarisation.

L'objectif de cette étude n'est pas d'examiner directement les causes du décrochage culturel. Il est de vérifier l'hypothèse selon laquelle les élèves francophones quittent leurs écoles parce qu'ils s'y sentent moins bien que les anglophones dans leurs écoles, parce que leur situation est différente de celle des élèves anglophones. Il nous semble important d'éliminer cette hypothèse avant de concentrer les analyses sur les causes et sur les modes de la circulation de l'idéologie pro-anglaise vers laquelle se tournent les jeunes qui se déplacent vers les écoles anglaises ou les parents de ces jeunes. Si, en effet, on ne note pas de différence entre les élèves selon qu'ils sont francophones ou anglophones, quant à la façon dont ils se représentent leur milieu, aux expériences scolaires, quant aux sentiments qui sont liés à la transition en général, cela signifiera qu'il importe de concentrer les recherches sur l'idéologie pro-anglaise.

\section{MÉthodologie}

Pour procéder à ces comparaisons, nous disposons des données d'une enquête qui a effectivement porté sur le phénomène de la transition. Ces données ont été recueillies auprès de jeunes, de parents et d'éducateurs entre les mois d'octobre et de décembre 2008. Ces individus étaient invités à participer à des discussions de groupe animées par des membres de l'équipe de recherche. Les séances duraient deux heures. Vers la fin de ces séances, les participants remplissaient un questionnaire. Les étudiants devaient en plus encercler, sur une feuille, des visages qui traduisaient des émotions. Les groupes pour ces causeries ont été formés en divers endroits de l'Ontario (région du Grand Toronto, Ottawa, Windsor, Sudbury et sa région, Thunder-Bay et sa région, Hearst/ Kapuskasing) avec l'aide de la direction de plusieurs écoles. Les échanges avaient lieu en anglais ou en français selon la langue 
de l'école ${ }^{15}$. Suite aux discussions de groupe, quelques jeunes ont aussi participé à des entretiens (voir le Tableau 1).

Il faut avoir à l'esprit que la progression dans le système d'éducation n'est pas tout à fait la même pour les francophones et pour les anglophones. En effet, dans la mesure où le passage a pour corollaire un changement d'établissement, pour bon nombre de francophones, ce passage a lieu davantage de la sixième à la septième année que de la huitième à la neuvième année. Pour cette raison, l'échantillon francophone compte des élèves de sixième (44) et de huitième (36) qui, dans chaque cas, sont appelés à changer d'établissement. Cette stratification permet de vérifier s'il y a une différence entre les deux ensembles.

Nos analyses se concentrent sur les questionnaires qui ont été remplis par les élèves. À certains endroits, les résultats de ces analyses trouvent quelques illustrations dans les propos qui ont été recueillis.

\section{LES ÉLÈVES FRANCOPHONES ET LES ÉLÈVES ANGLOPHONES DEVANT LA TRANSITION}

L'échantillon des élèves qui se sont exprimés lors des discussions de groupe est composé de 176 anglophones et de 80 francophones (dont 43,2 \% de garçons chez les premiers et 41,3\%, chez les seconds). Ces nombres, dans des analyses simples, autorisent quelques comparaisons.

\subsection{Profil des deux échantillons}

\subsubsection{Le groupe auquel on appartient}

Si l'on demande aux élèves : "À quel(s) groupe(s) culturel(s) estimes-tu appartenir? " («To which cultural group(s) do you feel you belong? »), les réponses sont relativement nombreuses, cette question étant ouverte. Elles sont au nombre de 42 pour les anglophones et de 26 pour les francophones. Trente-cinq anglophones et 29 francophones fournissent des réponses multiples.

15. Pour de plus amples détails sur la collecte de données, on se référera au rapport de Kate Tilleczek, Simon Laflamme, Bruce Ferguson, Dara Roth Edney, Mélanie Girard, Dana Cudney, Siobhan Cardoso, ibid. 


\section{Tableau 1}

Distribution échantillonnale de l'enquête

\begin{tabular}{|c|c|c|c|c|}
\hline \begin{tabular}{|l|} 
Discussions de \\
groupe avec les élèves \\
anglophones
\end{tabular} & Lieu & $\begin{array}{l}\mathrm{n}^{\text {bre }} \text { de groupes } \\
\text { de discussion }\end{array}$ & $\mathrm{n}^{\text {bre }}$ de participants & $\mathrm{n}^{\text {bre }}$ d'entretiens \\
\hline & GR de Toronto & 11 & 77 & 16 \\
\hline & Ottawa & 2 & 20 & 5 \\
\hline & Windsor & 2 & 15 & 4 \\
\hline & Sudbury+ & 6 & 45 & 12 \\
\hline & Thunder Bay+ & 2 & 19 & 6 \\
\hline \multicolumn{2}{|c|}{ Sous-total pour les élèves anglophones } & 23 & 176 & 43 \\
\hline \multirow[t]{5}{*}{$\begin{array}{l}\text { Discussions de } \\
\text { groupe avec les élèves } \\
\text { francophones }\end{array}$} & Lieu & $\begin{array}{l}\mathrm{n}^{\text {bre }} \text { de groupes } \\
\text { de discussion }\end{array}$ & $\mathrm{n}^{\text {bre }}$ de participants & $\mathrm{n}^{\text {bre }}$ d'entretiens \\
\hline & GR de Toronto & 3 & 25 & 2 \\
\hline & Ottawa & 3 & 25 & 2 \\
\hline & Sudbury+ & 3 & 18 & 3 \\
\hline & $\begin{array}{l}\text { Moonbeam } \\
\& \text { Hearst }\end{array}$ & 2 & 12 & 2 \\
\hline \multicolumn{2}{|c|}{ Sous-total pour les élèves francophones } & 11 & 80 & 9 \\
\hline \multicolumn{2}{|c|}{ Total pour les élèves } & 34 & 256 & 52 \\
\hline \multirow[t]{6}{*}{\begin{tabular}{|l|} 
Discussions de \\
groupe avec les \\
parents anglophones
\end{tabular}} & Lieu & $\begin{array}{l}\mathrm{n}^{\text {bre }} \text { de groupes } \\
\text { de discussion }\end{array}$ & $\mathrm{n}^{\text {bre }}$ de participants & \\
\hline & GR de Toronto & 2 & 4 & \\
\hline & Ottawa & 2 & 9 & \\
\hline & Windsor & 1 & 2 & \\
\hline & Sudbury+ & 1 & 6 & \\
\hline & Thunder Bay+ & 1 & 3 & \\
\hline \multicolumn{2}{|c|}{ Sous-total pour les parents anglophones } & 7 & 24 & \\
\hline \multirow[t]{2}{*}{$\begin{array}{l}\text { Discussions de } \\
\text { groupe avec les } \\
\text { parents francophones }\end{array}$} & Lieu & $\begin{array}{l}\mathrm{n}^{\text {bre }} \text { de groupes } \\
\text { de discussion }\end{array}$ & $\mathrm{n}^{\text {bre }}$ de participants & \\
\hline & $\begin{array}{l}\text { Moonbeam \& } \\
\text { Hearst }\end{array}$ & 1 & 3 & \\
\hline \multicolumn{2}{|c|}{ Sous-total pour les parents francophones } & 1 & 3 & \\
\hline \multicolumn{2}{|c|}{ Total pour les parents } & 8 & 27 & \\
\hline \multirow[t]{6}{*}{\begin{tabular}{|l|} 
Discussions de \\
groupe avec les édu- \\
cateurs anglophones
\end{tabular}} & Lieu & $\begin{array}{l}\mathrm{n}^{\text {bre }} \text { de groupes } \\
\text { de discussion }\end{array}$ & $\mathrm{n}^{\text {bre }}$ de participants & \\
\hline & GR de Toronto & 3 & 9 & \\
\hline & Ottawa & 2 & 11 & \\
\hline & Windsor & 1 & 3 & \\
\hline & Thunder Bay+ & 2 & 8 & \\
\hline & Attawapiskat & 1 & 2 & \\
\hline \multicolumn{2}{|c|}{ Sous-total pour les éducateurs anglophones } & 9 & 33 & \\
\hline \multicolumn{2}{|c|}{ Sous-total pour les éducateurs francophones } & 0 & 0 & \\
\hline \multicolumn{2}{|c|}{ Total pour les éducateurs } & 9 & 33 & \\
\hline
\end{tabular}


Chez les anglophones, le référent Canadian est le plus fréquent (24) ; viennent ensuite ceux d'Indian (12) et d'Italian (11), puis ceux de Mexican (8) et de Chinese (8) ; 7 élèves disent n'appartenir à aucun groupe culturel ; les autres groupes ne sont jamais mentionnés plus de 5 fois (Catholic, English, German, Scottish). Chez les francophones, les réponses les plus communes sont Canadien français (25) et Canadien (14) ; elles comptent pour près de $50 \%$ des réponses ; les autres identifications ne comptent jamais, en valeur absolue, pour plus de 2, sauf pour les dénominations Libanais (5) et Blanc (4) ${ }^{16}$. Dans l'échantillon global, donc, le composant francophone est moins marqué du signe de la pluralité que l'ensemble anglophone ${ }^{17}$.

\subsubsection{Le pays de naissance}

Dans le cas des deux échantillons, la majorité des individus est née au Canada : 85,8 \% et 82,5\% pour les anglophones et les francophones respectivement. Il n'y a donc pas de différence notable sur ce plan ${ }^{18}$. Dans l'ensemble anglophone, les pays où l'on est né, quand il ne s'agit pas du Canada, sont au nombre de 7 : India (6), United States (4), China (4), Pakistan (3), Bangladesh (3), United Arab Emirates (2) et Philippines (1). Chez les francophones, on compte 8 pays autres que le Canada : France (3), Iran (2), Bangladesh (1), Liban (1), Congo (1), Autriche (1) Guatemala (1) et Burundi (1). En nombre absolu, il y a plus de jeunes chez les anglophones que chez les francophones qui sont nés à l'extérieur du Canada ; mais les pays d'origine ne constituent pas un ensemble plus homogène et plus restreint chez les uns que chez les autres.

Le questionnaire demandait aux jeunes qui sont nés à l'extérieur du Canada s'ils étaient ou non heureux dans leur pays de naissance. Les réponses possibles sont au nombre de quatre, allant de « pas du tout » (« not at all »), soit 1, à « très heureux »

16. On notera qu'aucun jeune ne s'est défini comme Franco-Ontarien.

17. $\chi_{(52)}^{2}=122,18 ; \mathrm{p}<0,001$.

18. $\chi_{\text {corrigé }}^{2}=0,24 ; \mathrm{p}=0,62$. 
(«very happy»), soit 4. Les réponses peuvent être traitées comme cardinales de sorte qu'il est permis d'en calculer les moyennes. Si l'on compare les tendances centrales selon que les jeunes ont répondu au questionnaire en anglais ou en français, on ne trouve pas de différence inférable. Les moyennes sont de 3,50 $(\mathrm{s}=0,67)$ et $3,77(\mathrm{~s}=0,44)$, ce qui traduit des états de bonheur ${ }^{19}$. Le questionnaire demandait aussi à ces élèves s'ils sont heureux au Canada. L'échelle est la même ; elle rend donc possible une comparaison de moyennes; cette comparaison montre à nouveau que les deux groupes, celui des anglophones et celui des francophones, doivent être considérés comme semblables ${ }^{20}$, la moyenne des premiers étant de 3,54 $(\mathrm{s}=0,66)$ et celle des seconds, de 3,75 $(\mathrm{s}=0,45)$. Entre ces deux moments, celui du vécu dans le pays d'origine et celui de la vie canadienne, l'état de bonheur est équivalent quand on le mesure pour les deux échantillons réunis ${ }^{21}$ : si elle se rapporte au passé, à l'étranger, la moyenne est de 3,62 ; pour maintenant, au Canada, elle est exactement la même. Ainsi, chez les francophones et les anglophones qui sont nés à l'extérieur du Canada, le bonheur est considéré comme relativement élevé en moyenne, pour les uns et pour les autres, maintenant comme auparavant.

\subsubsection{La discrimination}

«Penses-tu faire partie d'un groupe qui est traité injustement au Canada?» («Do you consider yourself to be a member of a group that is unfairly treated in Canada? »); ainsi est formulée une des questions qui sont posées aux jeunes. La réponse négative est nettement majoritaire : 91,9\% chez les anglophones et 94,4\% chez les francophones. Il n'y a pas de différence entre les deux échantillons ${ }^{22}$. Parmi les 13 élèves qui ont répondu « oui » dans l'ensemble anglophone, il y en a onze qui ont indiqué de façon

19. $\mathrm{t}_{(32,58)}=-1,43 ; \mathrm{p}=0,16$.

20. $\mathrm{t}_{(34)}=-0,98 ; \mathrm{p}=0,33$.

21. $\mathrm{t}_{(34)}=0,00 ; \mathrm{p}=1,00$.

22. $\chi_{\text {corrigé }}^{2}=0,16 ; \mathrm{p}=0,69$. 
crédible $^{23}$ le groupe qui souffre de discrimination et dont ils font partie. Ces groupes sont les suivants : Aboriginal, Asian, Muslim, Weight, Italian, Irish, Arabic, Brown ; aucun d'entre eux n'est signalé plus de deux fois. Deux des 4 francophones qui ont fourni une réponse affirmative ont mentionné chacun la population à laquelle ils appartiennent qui, de leur point de vue, n'est pas traitée avec justice : les Inuits et les Haïtiens.

Il y a donc peu de différence entre les francophones et les anglophones en ce que l'immense majorité n'a pas l'impression d'appartenir à un groupe qui soit traité de manière injuste. Les groupes qui sont relevés sont par ailleurs variés. Chez les anglophones, il ne semble pas y avoir d'estimation concentrée du sentiment de discrimination.

\subsubsection{La langue}

Le questionnaire interroge les élèves sur la langue qu'ils parlent à l'école. Cent soixante et onze anglophones et 80 francophones ont répondu à la question. Quatre anglophones seulement ont mentionné une langue autre que l'anglais : deux ont écrit Ojibwe, un, Punjabi, et un autre, French. Chez les francophones, 22 d'entre eux ont répondu anglais et 58 , français.

Comme on pouvait le prévoir, les deux échantillons diffèrent sur ce point. Il faut souligner la forte quantité de francophones qui déclarent parler anglais à l'école, bien que les motifs puissent être nombreux en fonction de la manière dont on a compris la question : a-t-on à l'esprit les cours de langue ? pense-t-on à la langue parlée en dehors de la salle de classe ? Mais il reste que la presque totalité des anglophones disent de façon nette parler anglais à l'école et que le comportement des francophones à l'égard du français n'est pas de même nature.

Le questionnaire sollicite davantage d'informations sur la langue. Entre autres, il demande aux jeunes quelle langue ils parlent « la plupart du temps » (« most of the time »), avec leurs parents.

23. Un élève a écrit Slave ; un autre a ignoré la question. 
Les deux échantillons ne sont pas semblables sur ce point ${ }^{24}$. Des chiffres sont particulièrement évidents de contraste : $83 \%$ des élèves des écoles anglaises répondent que c'est l'anglais qu'ils parlent normalement avec leurs parents ; 49,4\% des jeunes de l'échantillon francophone répondent que cette langue est le français. 1,2\% des jeunes des écoles anglaises parlent français avec leurs parents « most of the time»; $38 \%$ des élèves francophones parlent anglais avec leurs parents « la plupart du temps ». Chez les élèves anglophones, 15 autres langues sont mentionnées dont les fréquences sont toutes inférieures à 5 ; les francophones en indiquent 8 autres, aucune fréquence ne dépassant la valeur de 2 .

Ces chiffres montrent bien que les francophones vivent en contexte minoritaire autant qu'ils témoignent de l'importance de la langue anglaise.

\subsubsection{Le statut socio-économique de la famille d'origine}

Les mères des élèves de l'échantillon francophone sont légèrement avantagées en ce qui a trait à l'instruction ${ }^{25}: 57,7 \%$ d'entre elles ont un diplôme d'études universitaires pour $42,4 \%$ des mères des élèves anglophones ; pour le diplôme d'études collégiales, ces chiffres sont respectivement de $31 \%$ et $26,7 \%$. Entre les pères, que leur enfant étudie en anglais ou en français, il n'y a pas de différence $^{26}: 42,3 \%$ de ceux dont l'enfant est inscrit dans une école anglaise détiennent un diplôme universitaire pour 44,3\% des pères des élèves francophones ; au collégial, les statistiques, dans le même ordre, sont de $23,1 \%$ et $32,9 \%$. Plus de $65 \%$ des parents ont donc fait des études postsecondaires. Il faut attribuer ce chiffre élevé aux contraintes auxquelles a dû se soumettre l'équipe de recherche pour construire l'échantillon. Les obligations d'ordre éthique ont été à ce point rigoureuses qu'elles ont rendu difficile la participation des enfants des familles les moins favorisées. Tout enfant, en effet, ne pouvait faire entendre sa voix dans les

24. $\chi_{(22)}^{2}=121,21 ; \mathrm{p}<0,001$.

25. $\chi_{(4)}^{2}=10,95 ; \mathrm{p}<0,05$.

26. $\chi_{(4)}^{2}=6,87 ; \mathrm{p}=0,14$. 
groupes de discussion que si ses parents avaient pris le temps de lire une documentation élaborée, avaient signé des formulaires de consentement et les avaient envoyés à l'école.

$\mathrm{Si}$, donc, l'ensemble des parents est surinstruit par rapport à la population générale, dans nos échantillons, il y a peu de différence entre les deux environnements familiaux pour ce qui est de l'instruction des parents.

Cette forte scolarisation des pères et des mères se reflète dans l'esprit des enfants quand on leur demande d'estimer le niveau de richesse de leur famille par rapport à celui des autres élèves. Trois réponses sont possibles : «n'a pas assez d'argent» («does not have enough money »), « a assez d'argent » (« has enough money»), «a beaucoup d'argent» («has lots of money»). Deux jeunes refusent de répondre chez les anglophones et $16 \mathrm{chez}$ les francophones. Seulement 8 élèves, et exclusivement chez les anglophones, choisissent la réponse la moins favorable. En retirant du tableau les jeunes qui omettent de fournir une réponse, on trouve que la plupart des autres considèrent que leur famille a assez d'argent, soit 77,3\% des anglophones et 67,2\% des francophones ; environ $25 \%$ se jugent privilégiés, soit 17,8\% des anglophones et 32,8\% des francophones. Les deux ensembles sont ainsi plutôt comparables en ce que le sentiment de pauvreté y est rare ou exclu, mais ils diffèrent quelque peu puisque le sentiment d'être très bien nanti y est plus fréquent chez les francophones ${ }^{27}$. Il importe ici de souligner que ce qui est décrit, c'est l'échantillon, et non la population dont il est issu, la sélection des individus n'ayant pas été faite sur un mode purement aléatoire.

Les échantillons comprennent en surnombre des élèves qui proviennent des familles privilégiées. Soit. Pour la suite des analyses, la question qui se pose n'est pas de savoir si le discours pro-anglais est plus commun dans les milieux francophones les moins instruits que dans les plus instruits. Cette question pertinente devra être examinée dans une autre recherche. La question que doit se poser le lecteur est la suivante : si l'étude avait compté

27. $\chi_{(2)}^{2}=8,48 ; \mathrm{p}<0,05$. 
une plus grande proportion d'élèves des familles les moins favorisées, y aurait-il eu plus de différence entre les francophones et les anglophones?

\subsection{Les amis}

Les amis sont-ils importants ? Pour le découvrir, on a demandé aux jeunes « à quel point est-ce important pour toi d'avoir des amis? » («How important are your friends to you? »). La question s'est rapportée à diverses conditions. À cette question, on peut répondre sur une échelle de Likert à quatre niveaux allant de 1 , soit « pas du tout » (« not at all »), à 4, soit « très important » («very important »). On peut alors calculer des moyennes et les comparer en fonction d'échantillons (voir le Tableau 2). Si la condition est « après l'école et les fins de semaine » («after school and week ends »), l'importance des amis est manifeste : la moyenne, pour les anglophones comme pour les francophones, est supérieure à 3, au-delà, donc, d'une valeur qui serait « assez important» («fairly important»). Si l'on invite les jeunes à se référer à la condition qui correspond à « pendant la journée scolaire » («during the school day»), les moyennes sont même plus élevées. Pour aucun de ces cas il n'y a de différence significative entre francophones en anglophones.

\section{Tableau 2}

Différence de moyennes pour l'importance des amis dans diverses circonstances

selon que l'élève étudie dans une école anglophone ou francophone

\begin{tabular}{|c|c|c|c|c|c|c|}
\hline \multicolumn{7}{|c|}{$\begin{array}{l}\text { (Réponses en français : } 1=\text { pas du tout } ; 4=\text { très important) } \\
\text { (Réponses en anglais : } 1=\text { not at all } ; 4=\text { very important })\end{array}$} \\
\hline \multirow{2}{*}{$\begin{array}{l}\text { À quel point est-ce important } \\
\text { pour toi d'avoir des amis... } \\
\text { (How important are } \\
\text { your friends to you...) }\end{array}$} & & Langu & e l'école & \multirow[t]{2}{*}{$\mathrm{t}$} & \multirow[t]{2}{*}{$\mathrm{D}$} & \multirow[t]{2}{*}{$\mathrm{p}<0,05$} \\
\hline & & anglai & français & & & \\
\hline \multirow{2}{*}{$\begin{array}{l}\text { après l'école et la fin } \\
\text { de semaine? } \\
\text { (after school and weekends?) }\end{array}$} & $\overline{\mathrm{X}}$ & 3,34 & 3,40 & \multirow[t]{2}{*}{$-0,60$} & \multirow[t]{2}{*}{250} & \multirow[t]{2}{*}{ non } \\
\hline & $\mathrm{S}$ & 0,72 & 0,71 & & & \\
\hline \multirow{2}{*}{$\begin{array}{l}\text { pendant la journée scolaire? } \\
\text { (during the school day?) }\end{array}$} & $\overline{\mathrm{X}}$ & 3,57 & 3,65 & \multirow[t]{2}{*}{$-0,96$} & \multirow[t]{2}{*}{250} & \multirow[t]{2}{*}{ non } \\
\hline & $\mathrm{S}$ & 0,63 & 0,56 & & & \\
\hline
\end{tabular}


Lisons ces deux échanges entre l'animatrice et des élèves :

Élève ${ }^{28}$ : $\quad$ Mais, les amis comme... je ne serais pas capable de le faire [passage au secondaire] sans eux. Des fois, s'ils vont te dire que : «Ok, moi, je m'en vais en arts plastiques », puis, moi, je dis : « Non, moi, je vais aller en musique », je vais garder musique ; mais je vais être quand même amie avec eux durant le dîner.

Animatrice : Ok.

Élève : $\quad$ C'est, comme, surtout ce que, moi, je veux faire ; mais il ne faut pas que je perde mes amis en le faisant.

$$
* * *
$$

Animatrice : Quand vous pensez à votre choix d'une école secondaire, parce que j'imagine que c'est un choix que vous avez à faire : à quelle école vous voulez aller. Ce sont quoi les facteurs qui sont les plus importants? Qui influencent votre décision? Oui, [nom de l'élève]?

Élève ${ }^{29}$ : $\quad$ Si j'ai un bon groupe d'amis, je vais aller à cette école-là. Je ne suis pas pour choisir une école où je sais que je n'ai pas d'amis et être tout seul dans mon coin, et ça va être difficile d'en faire d'autres. Tu sais : tu arrives au milieu de l'année ; c'est pas tout le monde qui vont vouloir être ton ami, parce qu'ils ne te connaissent pas depuis le primaire ; alors qu'ici, j'avais déjà des amis du primaire ; alors j'ai embarqué avec eux ; je me suis fait d'autres amis ; alors c'est pour cela que je choisis mon école avec qui, mes amis.

\subsection{Soi et les autres}

Le questionnaire propose aux jeunes de se comparer aux autres sur divers objets comme la taille ou la responsabilité. Les objets ne sont pas toujours les mêmes pour les francophones et pour les

28. Élève \$2YC2FF078C.

29. Élève \$2YC2FF081C. 
anglophones, et le type de variable n'est pas non plus identique : les variables sont cardinales $(1=$ strongly disagree et $4=$ strongly agree) dans le questionnaire anglophone et nominales (oui et non) dans le questionnaire francophone.

Les jeunes des écoles anglophones (voir le Tableau 3), dans notre échantillon, tendent à se sentir plus responsables, plus mûrs que leurs camarades de classe. Sur les autres aspects, notamment en ce qui a trait à l'apparence physique, les opinions tendent à être plus partagées. Mais les moyennes, inférieures à 3, souvent tout près de 2 , et les écarts types, toujours à proximité de 1 , révèlent que plusieurs jeunes se sentent différents des autres

\section{Tableau 3}

Estimation de soi sur divers objets par comparaison aux autres élèves de la classe Échantillon anglophone

\begin{tabular}{|c|c|c|c|c|}
\hline \multicolumn{5}{|c|}{$\begin{array}{l}\text { Moyenne }(\overline{\mathrm{x}}), \text { médiane }(\mathrm{Md}), \text { mode }(\mathrm{Mo}) \text { et écart type }(\mathrm{s}) \\
(1=\text { strongly disagree et } 4=\text { strongly agree }) \\
\quad 161 \leq \mathrm{n} \leq 174\end{array}$} \\
\hline Objet de la comparaison & \multirow[t]{2}{*}{$\overline{\mathrm{x}}$} & \multirow[t]{2}{*}{$\mathrm{Md}$} & \multirow[t]{2}{*}{ Mo } & \multirow[t]{2}{*}{$\mathrm{s}$} \\
\hline Compared to your class mates this year, do you... & & & & \\
\hline look taller? & 2,51 & 3,00 & 3 & 0,96 \\
\hline look shorter? & 2,18 & 2,00 & 2 & 1,04 \\
\hline look younger? & 2,10 & 2,00 & 2 & 0,81 \\
\hline look older? & 2,66 & 3,00 & 3 & 0,81 \\
\hline feel like you are good looking? & 2,71 & 3,00 & 3 & 0,83 \\
\hline feel more grown up? & 2,98 & 3,00 & 3 & 0,67 \\
\hline feel more responsible? & 3,20 & 3,00 & 3 & 0,72 \\
\hline
\end{tabular}

Les données de l'échantillon francophone vont dans le même sens, même si leur forme est statistiquement différente (voir le Tableau 4). Les jeunes, là, expriment dans une forte proportion leur sentiment d'être plus responsables que leurs camarades de classe, mais ils ne se considèrent pas pour autant plus adultes. On note par ailleurs des proportions non négligeables d'élèves qui estiment avoir l'air plus grand, ou plus petit, ou plus jeune, ou plus vieux que les autres. 
Tableau 4

Estimation de soi sur divers objets

par comparaison aux autres élèves de la classe

Échantillon francophone

\begin{tabular}{|c|c|c|c|c|}
\hline \multicolumn{5}{|l|}{ (Réponse en centièmes pour les oui et les non) } \\
\hline Objet de la comparaison & \multicolumn{2}{|c|}{ Réponse } & \multirow[t]{2}{*}{ Total } & \multirow[t]{2}{*}{$\mathrm{n}^{\text {bre }}$} \\
\hline Par rapport à tes camarades de classe de cette année... & oui & non & & \\
\hline as-tu l'air plus grand(e)? & 0,49 & 0,51 & 1,00 & 76 \\
\hline as-tu l'air plus petit(e)? & 0,39 & 0,61 & 1,00 & 71 \\
\hline as-tu l'air plus jeune? & 0,34 & 0,66 & 1,00 & 68 \\
\hline as-tu l'air plus vieux ou plus vieille? & 0,31 & 0,69 & 1,00 & 68 \\
\hline as-tu l'impression d'être plus adulte? & 0,29 & 0,71 & 1,00 & 69 \\
\hline as-tu l'impression d'être plus responsable? & 0,76 & 0,24 & 1,00 & 71 \\
\hline
\end{tabular}

\subsection{Manière dont on se sent à l'école}

Les élèves sont invités à prendre position sur une série d'énoncés dans lesquels est exprimé un sentiment à l'égard de soi à l'école. En prenant position, les élèves choisissent une valeur entre 1 et 4, soit entre « pas du tout d'accord » (« strongly disagree ») et « tout à fait d'accord » (« strongly agree »). Il s'agit d'échelles de Likert avec lesquelles les opérations arithmétiques sont permises. Entre les francophones et les anglophones, à se fier aux tests d'hypothèses (voir le Tableau 5), il y a quelques différences : les francophones se sentent moins étrangers à l'école que les anglophones, ont plus l'impression de s'y faire facilement des amis, s'y sentent plus à leur aise, s'y sentent moins seuls, ont plus l'impression de faire partie du groupe, hésitent moins à y aller et s'y ennuient moins. Mais ces tests sont suspects : s'ils traduisent des tendances possibles, ils ne rendent pas compte de grandes différences entre les deux populations. La différence de moyennes la plus marquée est de 0,51 sur une échelle de 4 ; elle a trait à l'ennui qu'on éprouve à l'école. Les autres différences qu'il est permis de ne pas attribuer au hasard se situent entre 0,24 et 0,43 . Il faut surtout souligner, dans tous ces résultats, le fait que les moyennes, même si elles ne dépeignent pas un état idéal, décrivent malgré tout des opinions positives. Les moyennes sont plus proches du désaccord que de l'accord pour un énoncé comme « mon école est un endroit où j'ai l'impression d'être un étranger 


\section{Tableau 5}

Différence de moyennes pour la manière dont on se sent à l'école à l'égard de divers aspects

selon que l'élève étudie dans une école anglophone ou francophone

\begin{tabular}{|c|c|c|c|c|c|c|}
\hline \multicolumn{7}{|c|}{$\begin{array}{l}\text { (Réponses en français : } 1=\text { pas du tout d'accord ; } 4 \text { = tout à fait d'accord) } \\
\text { (Réponses en anglais : } 1=\text { strongly disagree } ; 4=\text { strongly agree) }\end{array}$} \\
\hline \multirow{2}{*}{$\begin{array}{l}\text { Mon école est un endroit où... } \\
\text { (My school is a place where...) }\end{array}$} & & \multirow{2}{*}{\multicolumn{2}{|c|}{$\begin{array}{l}\text { Langue de l'école } \\
\text { anglais français }\end{array}$}} & \multirow[t]{2}{*}{$\mathrm{t}$} & \multirow[t]{2}{*}{ D } & \multirow[t]{2}{*}{$\mathrm{p}<0,05$} \\
\hline & & & & & & \\
\hline \multirow{2}{*}{$\begin{array}{l}\text { j'ai l'impression d'être } \\
\text { un étranger ou une étrangère } \\
\text { (Ifeel like an outsider } \\
\text { (or left out things)) }\end{array}$} & $\overline{\mathrm{x}}$ & 1,65 & 1,27 & \multirow[t]{2}{*}{4,26} & \multirow[t]{2}{*}{200,57} & \multirow[t]{2}{*}{ oui } \\
\hline & S & 0,81 & 0,59 & & & \\
\hline \multirow{2}{*}{$\begin{array}{l}\text { je me fais facilement des amis } \\
\text { (I make friends easily) }\end{array}$} & $\overline{\mathrm{x}}$ & 3,07 & 3,37 & \multirow[t]{2}{*}{$-3,09$} & \multirow[t]{2}{*}{138,13} & \multirow[t]{2}{*}{ oui } \\
\hline & S & 0,65 & 0,72 & & & \\
\hline \multirow{2}{*}{$\begin{array}{l}\text { je ne suis pas à l'aise et } \\
\text { je ne me sens pas à ma place } \\
\text { (I feel awkward and out of place) }\end{array}$} & $\bar{x}$ & 1,74 & 1,47 & \multirow[t]{2}{*}{2,44} & \multirow[t]{2}{*}{247} & \multirow[t]{2}{*}{ oui } \\
\hline & $\mathrm{S}$ & 0,82 & 0,70 & & & \\
\hline \multirow{2}{*}{$\begin{array}{l}\text { d'autres élèves ont l'air } \\
\text { de m'apprécier } \\
\text { (other students seem to like me) }\end{array}$} & $\bar{x}$ & 3,06 & 3,05 & \multirow[t]{2}{*}{0,86} & \multirow[t]{2}{*}{248} & \multirow[t]{2}{*}{ non } \\
\hline & $\mathrm{S}$ & 0,55 & 0,66 & & & \\
\hline \multirow{2}{*}{$\begin{array}{l}\text { je me sens seul(e) } \\
\text { (Ifeel lonely) }\end{array}$} & $\bar{x}$ & 1,62 & 1,38 & \multirow[t]{2}{*}{2,35} & \multirow[t]{2}{*}{249} & \multirow[t]{2}{*}{ oui } \\
\hline & $\mathrm{S}$ & 0,76 & 0,73 & & & \\
\hline \multirow{2}{*}{$\begin{array}{l}\text { j'ai l'impression de faire } \\
\text { partie du groupe } \\
\text { (Ifeel like I belong) }\end{array}$} & $\bar{x}$ & 2,92 & 3,22 & \multirow[t]{2}{*}{$-2,96$} & \multirow[t]{2}{*}{249} & \multirow[t]{2}{*}{ oui } \\
\hline & $\mathrm{S}$ & 0,72 & 0,73 & & & \\
\hline \multirow{2}{*}{$\begin{array}{l}\text { je ne veux pas aller } \\
\text { (I do not want to go) }\end{array}$} & $\bar{x}$ & 2,19 & 1,76 & \multirow[t]{2}{*}{3,51} & \multirow[t]{2}{*}{248} & \multirow[t]{2}{*}{ oui } \\
\hline & $\mathrm{S}$ & 0,93 & 0,82 & & & \\
\hline \multirow{2}{*}{$\begin{array}{l}\text { je m'ennuie souvent } \\
\text { (I often feel bored) }\end{array}$} & $\overline{\mathrm{x}}$ & 2,49 & 1,97 & \multirow[t]{2}{*}{4,21} & 248 & oui \\
\hline & $\mathrm{S}$ & 0,88 & 0,90 & & & \\
\hline
\end{tabular}

ou une étrangère »; elles sont plus proches de l'accord pour la proposition « ... d'autres élèves ont l'air de m'apprécier »; plus près du désaccord quand les jeunes lisent « ... je me sens seul(e)».

\section{5. Évaluation de l'école}

Quel jugement les élèves portent-ils sur leur école? Pour le savoir, nous les avons invités à se prononcer sur neuf caractéristiques, comme la taille des bâtiments ou le soutien pour les élèves qui sont en difficulté. Pour émettre leur jugement, ils pouvaient choisir entre quatre réponses : « mauvaise » («not good at all »), « pas très bonne » («not so good»), « bonne » («good») ou « très bonne » 
(«very good »). De mauvaise à très bonne, les valeurs vont de 1 à 4 , ce qui, à nouveau, rend possible le calcul de moyennes.

Si l'on compare les réponses des francophones à celles des anglophones, il n'y a pas en réalité de différences significatives (voir le Tableau 6). Les tests n'en repèrent que deux : 1'une, pour le nombre d'élèves par classe, où les francophones sont un peu plus positifs, et, l'autre, pour l'accès à des aires ouvertes disponibles pour les élèves, où ce sont les anglophones qui semblent le plus satisfaits, mais à peine. On peut donc considérer que les deux ensembles posent un regard semblable sur leurs écoles. Ce regard est quelque peu critique, sans être sombre. Il laisse entendre qu'il y a place à l'amélioration sur des points comme la taille des bâtiments, l'accès à des espaces libres où les jeunes peuvent tout simplement être ensemble, le nombre d'élèves par classe ou l'appui dans la sélection des cours.

\subsection{Impression sur la diversité de l'école}

Les jeunes sont invités à estimer le niveau de diversité culturelle, raciale et linguistique des élèves et des enseignants de leur école ${ }^{30}$. Ils le font sur une échelle de Likert qui va de 1, «pas du tout» («not at all »), à 4, « très » (« very »). Il n'y a pas de différence significative des moyennes dans le jugement ni sur les élèves ${ }^{31}$ ni sur les enseignants ${ }^{32}$. Dans l'estimation de la diversité de la population étudiante, les moyennes sont d'environ 2,75 (avec des écarts types qui avoisinent la valeur de 1) ; cela signifie que les jeunes, dans l'ensemble, constatent un certain niveau de diversité. Quand l'opinion porte sur les enseignants, les moyennes sont plus faibles, se situant quelque peu au-dessus de la valeur de 2 , ce qui donne à entendre que, selon les élèves, le personnel enseignant est un peu moins hétérogène que ne l'est la population étudiante.

30. La population étudiante de ton école est-elle diversifiée (culture/ethnie/ langue) ? (How culturally/racially/linguistically diverse are the students at your school ?) La personnel scolaire et enseignant de ton école est-il diversifié (culture/ ethnie/langue)? (How culturally/racially/linguistically diverse are the teachers and staff at your school?)

31. $\mathrm{t}_{(191)}=0,60 ; \mathrm{p}=0,55$.

32. $\mathrm{t}_{(69,96)}=1,32 ; \mathrm{p}=0,19$. 


\section{Tableau 6}

Différence de moyennes pour l'évaluation de l'école à l'égard de divers critères

selon que l'élève étudie dans une école anglophone ou francophone

\begin{tabular}{|c|c|c|c|c|c|c|}
\hline \multicolumn{7}{|c|}{$\begin{array}{l}\text { (Réponses en français : } 1=\text { mauvaise } ; 4=\text { très bonne) } \\
\text { (Réponses en anglais : } 1=\text { not good at all } ; 4=\text { very good) }\end{array}$} \\
\hline \multirow[b]{2}{*}{$\begin{array}{l}\text { Comment trouves-tu ton école } \\
\text { actuellement par rapport aux } \\
\text { critères suivants? (How would } \\
\text { you rate the school you are } \\
\text { currently attending in terms of } \\
\text { the following?) }\end{array}$} & & \multicolumn{2}{|c|}{ Langue de l'école } & \multirow[t]{2}{*}{$\mathrm{t}$} & \multirow[t]{2}{*}{$\mathrm{D}$} & \multirow[t]{2}{*}{$\mathrm{p}<0,05$} \\
\hline & & anglais & français & & & \\
\hline \multirow{2}{*}{$\begin{array}{l}\text { Taille des bâtiments } \\
\text { (Building size) }\end{array}$} & $\overline{\mathrm{x}}$ & 2,68 & 2,77 & \multirow{2}{*}{$-0,77$} & \multirow[t]{2}{*}{226} & \multirow[t]{2}{*}{ non } \\
\hline & $\mathrm{S}$ & 0,73 & 0,68 & & & \\
\hline \multirow{2}{*}{$\begin{array}{l}\text { Nombre d'élèves par classe } \\
\text { (Number of students per class) }\end{array}$} & $\overline{\mathrm{x}}$ & 2,74 & 3,05 & \multirow[t]{2}{*}{$-2,71$} & \multirow[t]{2}{*}{229} & \multirow[t]{2}{*}{ oui } \\
\hline & $\mathrm{S}$ & 0,75 & 0,78 & & & \\
\hline \multirow{2}{*}{$\begin{array}{l}\text { Atmosphère à l'école } \\
\text { (School attitude/spirit) }\end{array}$} & $\overline{\mathrm{X}}$ & 3,02 & 2,94 & \multirow[t]{2}{*}{0,70} & \multirow[t]{2}{*}{103,3} & \multirow[t]{2}{*}{ non } \\
\hline & $\mathrm{S}$ & 0,80 & 0,64 & & & \\
\hline \multirow{2}{*}{$\begin{array}{l}\text { Appui dans ta sélection de cours } \\
\text { (Supporting course choice) }\end{array}$} & $\overline{\mathrm{x}}$ & 2,70 & 2,81 & \multirow[t]{2}{*}{$-0,59$} & \multirow[t]{2}{*}{182} & \multirow[t]{2}{*}{ non } \\
\hline & $\mathrm{S}$ & 0,86 & 1,06 & & & \\
\hline \multirow{2}{*}{$\begin{array}{l}\text { Sécurité } \\
\text { (Safety) }\end{array}$} & $\overline{\mathrm{X}}$ & 3,10 & 3,13 & \multirow[t]{2}{*}{$-0,35$} & \multirow[t]{2}{*}{225} & \multirow[t]{2}{*}{ non } \\
\hline & $\mathrm{S}$ & 0,68 & 0,66 & & & \\
\hline \multirow{2}{*}{$\begin{array}{l}\text { Utilisation de tests équitables } \\
\text { (Using fair tests) }\end{array}$} & $\overline{\mathrm{x}}$ & 2,91 & 2,83 & \multirow[t]{2}{*}{0,68} & \multirow[t]{2}{*}{71,34} & \multirow[t]{2}{*}{ non } \\
\hline & $\mathrm{S}$ & 0,65 & 0,81 & & & \\
\hline \multirow{2}{*}{$\begin{array}{l}\text { Accès à des activités } \\
\text { parascolaires (Student access to } \\
\text { extra-curricular activities) }\end{array}$} & $\bar{x}$ & 3,02 & 2,82 & \multirow[t]{2}{*}{1,42} & \multirow[t]{2}{*}{222} & \multirow[t]{2}{*}{ non } \\
\hline & $\mathrm{S}$ & 0,89 & 0,90 & & & \\
\hline \multirow{2}{*}{$\begin{array}{l}\text { Accès à des aires ouvertes où les } \\
\text { élèves peuvent simplement } \\
\text { " être ensemble » (Open areas } \\
\text { for students to "hang out") }\end{array}$} & $\overline{\mathrm{x}}$ & 2,62 & 2,33 & 2,03 & 221 & oui \\
\hline & $\mathrm{S}$ & 0,88 & 0,75 & & & \\
\hline Soutien pour les élèves qui ont & $\overline{\mathrm{x}}$ & 2,94 & 2,71 & 1,46 & & \\
\hline $\begin{array}{l}\text { des difficultés à l'école } \\
\text { (Support for students having } \\
\text { trouble in school) }\end{array}$ & $\mathrm{S}$ & 0,80 & 1,05 & & 68,22 & non \\
\hline
\end{tabular}

\subsection{Opinion sur les enseignants}

Si l'on demande aux élèves de juger de leurs enseignants en fonction de divers aspects, on ne trouve pas de différence entre les anglophones et les francophones. À côté de ces aspects, les élèves trouvent quatre possibilités de réponses : « mauvais » («not good 
at all »), « pas très bon » («not so good 》), «bon » (« good») ou « très bon » (« very good »); il s'agit, en réalité, d'une échelle à quatre niveaux; et, par conséquent, les opérations arithmétiques sont permises. Seulement 1 test d'hypothèse sur 9 signale une inégalité de moyennes dont on pourrait penser qu'elle n'est pas attribuable au hasard (voir le Tableau 7) ; il porte sur l'énoncé « [mes enseignants] m'aident à prendre des décisions par rapport à l'école secondaire» («Helping you with high school decisions »). La différence n'est que de 0,26, sur une échelle de quatre. Elle ne mérite donc pas qu'on s'y attarde. On peut donc affirmer que les élèves, qu'ils soient francophones ou anglophones, perçoivent leurs enseignants de la même manière. Les moyennes sont toutes à proximité de la valeur de 3 , ce qui indique un jugement plutôt favorable quoique empreint de réserves. Les élèves considèrent que leurs enseignants sont plutôt enclins à les appuyer dans leur apprentissage, à rendre l'apprentissage amusant, à se soucier d'eux, à se soucier de leurs amis, à les laisser travailler avec leurs amis; ils estiment même que ces enseignants tendent à être accessibles ; mais ils ne s'agit pas de positions unanimes, et les enthousiasmes sont rares.

Deux propositions ont un intérêt particulier : « [les enseignants] donnent des renseignements sur l'école secondaire » (« Giving information on high school») et « [les enseignants] m'aident à prendre des décisions par rapport à l'école secondaire (« Helping you with high school decisions »). Les moyennes révèlent ici que les élèves ont l'impression d'être exposés à des enseignants qui tendent à les informer sur l'école secondaire.

\subsection{Encouragement des parents et des enseignants}

L'immense majorité des jeunes considèrent que leurs parents (ou tuteurs) les « encouragent à bien travailler à l'école » (« encourage to do well in school »). La réponse est positive pour 97,6 \% des anglophones et $96,8 \%$ des francophones, et il n'y a évidemment pas de différence entre les uns et les autres ${ }^{33}$.

33. $\chi_{\text {corrige }}^{2}=0,00 ; p=1,00$. 
Tableau 7

Différence de moyennes pour l'évaluation des enseignants

à l'égard de divers critères

selon que l'élève étudie dans une école anglophone ou francophone

\begin{tabular}{|c|c|c|c|c|c|c|}
\hline \multicolumn{7}{|c|}{$\begin{array}{l}\text { (Réponses en français : } 1=\text { mauvais } ; 4=\text { très bon) } \\
\text { (Réponses en anglais : } 1=\text { not good at all } ; 4=\text { very good })\end{array}$} \\
\hline \multirow{2}{*}{$\begin{array}{l}\text { Comment trouves-tu tes } \\
\text { enseignant(e)s par rapport aux } \\
\text { critères suivants? (How would } \\
\text { you rate your teachers in terms } \\
\text { of the following?) }\end{array}$} & & \multicolumn{2}{|c|}{ Langue de l'école } & \multirow[t]{2}{*}{$\mathrm{t}$} & \multirow[t]{2}{*}{$\mathrm{D}$} & \multirow[t]{2}{*}{$\mathrm{p}<0,05$} \\
\hline & & anglais & rançais & & & \\
\hline \multirow{2}{*}{$\begin{array}{l}\text { Ils appuient mon apprentissage } \\
\text { (Supporting your learning) }\end{array}$} & $\overline{\mathrm{x}}$ & 3,11 & 3,16 & \multirow[t]{2}{*}{$-0,45$} & \multirow[t]{2}{*}{223} & \multirow[t]{2}{*}{ non } \\
\hline & $\mathrm{s}$ & 0,67 & 0,47 & & & \\
\hline \multirow{2}{*}{$\begin{array}{l}\text { Ils donnent des renseignements } \\
\text { sur l'école secondaire } \\
\text { (Giving information on high school) }\end{array}$} & $\overline{\mathrm{x}}$ & 3,01 & 2,86 & \multirow[t]{2}{*}{1,19} & \multirow[t]{2}{*}{224} & \multirow[t]{2}{*}{ non } \\
\hline & $\mathrm{s}$ & 0,80 & 0,78 & & & \\
\hline \multirow{2}{*}{$\begin{array}{l}\text { Ils m'aident à prendre des } \\
\text { décisions par rapport à l'école } \\
\text { secondaire (Helping you with } \\
\text { high school decisions) }\end{array}$} & $\overline{\mathrm{x}}$ & 2,89 & 2,63 & \multirow[t]{2}{*}{2,01} & \multirow[t]{2}{*}{223} & \multirow[t]{2}{*}{ oui } \\
\hline & $\mathrm{s}$ & 0,81 & 0,76 & & & \\
\hline \multirow{2}{*}{$\begin{array}{l}\text { Ils rendent l'apprentissage } \\
\text { amusant (Making learning fun) }\end{array}$} & $\overline{\mathrm{X}}$ & 2,72 & 2,66 & \multirow[t]{2}{*}{0,41} & \multirow[t]{2}{*}{224} & \multirow[t]{2}{*}{ non } \\
\hline & $\mathrm{S}$ & 0,94 & 0,94 & & & \\
\hline \multirow{2}{*}{$\begin{array}{l}\text { Ils se soucient de moi } \\
\text { (Caring about you) }\end{array}$} & $\overline{\mathrm{x}}$ & 3,02 & 2,82 & \multirow[t]{2}{*}{1,65} & \multirow[t]{2}{*}{224} & \multirow[t]{2}{*}{ non } \\
\hline & $\mathrm{S}$ & 0,77 & 0,75 & & & \\
\hline \multirow{2}{*}{$\begin{array}{l}\text { Ils se soucient de mes amis } \\
\text { (Caring about your friends) }\end{array}$} & $\overline{\mathrm{x}}$ & 2,90 & 2,84 & \multirow[t]{2}{*}{0,52} & \multirow[t]{2}{*}{224} & \multirow[t]{2}{*}{ non } \\
\hline & $\mathrm{S}$ & 0,77 & 0,77 & & & \\
\hline \multirow{2}{*}{$\begin{array}{l}\text { Ils sont de bon(ne)s } \\
\text { enseignant }(\mathrm{e}) \mathrm{s} \\
(\text { Being good teachers })\end{array}$} & $\overline{\mathrm{X}}$ & 3,14 & 3,14 & \multirow[t]{2}{*}{$-0,05$} & \multirow[t]{2}{*}{223} & \multirow[t]{2}{*}{ non } \\
\hline & $\mathrm{s}$ & 0,74 & 0,68 & & & \\
\hline \multirow{2}{*}{$\begin{array}{l}\text { Ils me laissent travailler avec } \\
\text { mes amis en classe (Letting your } \\
\text { friends work with you in class) }\end{array}$} & $\overline{\mathrm{x}}$ & 2,73 & 2,51 & 1,65 & 223 & non \\
\hline & $\mathrm{S}$ & 0,85 & 0,79 & & & \\
\hline Il est facile de leur parler & $\overline{\mathrm{X}}$ & 2,94 & 2,88 & 0,42 & 222 & non \\
\hline$($ Easy to talk to $)$ & $\mathrm{s}$ & 0,89 & 0,88 & & & \\
\hline
\end{tabular}

Si la question est posée en référence aux enseignants, la majorité des réponses est aussi positive, mais dans une moindre mesure. C'est « oui » pour 83,2\% des élèves francophones et 75,9\% des anglophones. Les «non » sont exceptionnels : 4 (2,3\%) seulement chez les anglophones, 0 chez les francophones. La différence entre 
la distribution des réponses pour les parents et celle qui vaut pour les enseignants vient peut-être du fait que, dans ce second cas, les élèves ont une troisième possibilité de réponse : "parfois 》 (« sometimes »); $14,5 \%$ des élèves anglophones et $24,1 \%$ des élèves francophones optent pour cette réponse. Il n'y a pas de différence significative entre les deux groupes linguistiques ${ }^{34}$.

À l'évidence, donc, les jeunes ont le sentiment d'être entourés de parents et d'enseignants qui les encouragent à bien travailler.

\subsection{Importance de l'école secondaire dans l'environnement de l'élève}

Les jeunes eux-mêmes croient-ils que les études secondaires sont importantes? Trouvent-ils qu'elles sont importantes aux yeux de leurs parents ? et de leurs amis ? Les questions leur ont été posées. Ils ont pu fournir des réponses sur une échelle de Likert à quatre niveaux dont les pôles sont « pas du tout importantes » (« not at all important »), 1, et « très importantes » («very important »), 4. L'analyse montre de façon manifeste (voir le Tableau 8) que les jeunes se représentent de façon hautement positive les études secondaires et que, dans leur optique, il en va de même pour leurs parents : les moyennes frôlent la valeur absolue de 4, et il n'y a pas, sous ces angles, de variations entre les francophones et les anglophones. Si l'on s'en remet à leur opinion, leurs amis valorisent moins, comme groupe, ces études secondaires, et c'est encore plus vrai pour les francophones que pour les anglophones, quoique l'écart ne soit pas alarmant.

\subsection{Aide pour le travail scolaire}

"As-tu l'impression d'avoir besoin d'aide pour ton travail scolaire ou tes devoirs?» («Do you feel you need help with your schoolwork/assignments?»). La question a été posée en ces termes aux élèves. La répartition des réponses n'est pas tout à fait la même pour les anglophones et pour les francophones ${ }^{35}$ : aucun franco-

34. $\chi_{(2)}^{2}=2,31 ; \mathrm{p}=0,32$.

35. $\chi_{(2)}^{2}=7,23 ; \mathrm{p}<0,05$. 


\section{Tableau 8}

Différence de moyennes pour l'importance de l'école secondaire pour diverses populations

selon que l'élève étudie dans une école anglophone ou francophone

\begin{tabular}{|c|c|c|c|c|c|c|}
\hline \multicolumn{7}{|c|}{$\begin{array}{l}\text { (Réponses en français : } 1=\text { pas du tout important } ; 4=\text { très important) } \\
\text { (Réponses en anglais : } 1=\text { not at all important } ; 4=\text { very important) }\end{array}$} \\
\hline \multirow{2}{*}{$\begin{array}{l}\text { Quelle est l'importance d'une } \\
\text { éducation secondaire pour... } \\
\text { (How important is a high } \\
\text { school education to...) }\end{array}$} & \multicolumn{3}{|c|}{ ngue de l'école } & \multirow[t]{2}{*}{$\mathrm{t}$} & \multirow[t]{2}{*}{$\mathrm{D}$} & \multirow[t]{2}{*}{$\mathrm{p}<0,05$} \\
\hline & & anglais & français & & & \\
\hline \multirow[t]{2}{*}{ toi ? (you?) } & $\overline{\mathrm{X}}$ & 3,88 & 3,84 & \multirow[t]{2}{*}{0,50} & \multirow[t]{2}{*}{203} & \multirow[t]{2}{*}{ non } \\
\hline & $\mathrm{S}$ & 0,35 & 0,45 & & & \\
\hline \multirow{2}{*}{$\begin{array}{l}\text { tes parents ou tuteurs? } \\
\text { (your parent(s) or guardians(s)?) }\end{array}$} & $\overline{\mathrm{x}}$ & 3,92 & 3,88 & \multirow[t]{2}{*}{0,66} & \multirow[t]{2}{*}{203} & \multirow[t]{2}{*}{ non } \\
\hline & $\mathrm{s}$ & 0,29 & 0,55 & & & \\
\hline \multirow[t]{2}{*}{ tes amis ? (your friends?) } & $\overline{\mathrm{X}}$ & 3,39 & 2,94 & \multirow[t]{2}{*}{3,60} & \multirow[t]{2}{*}{200} & \multirow[t]{2}{*}{ oui } \\
\hline & $\mathrm{S}$ & 0,61 & 0,88 & & & \\
\hline
\end{tabular}

phone ne répond oui alors que $9,4 \%$ des anglophones le font ; $25,7 \%$ des anglophones et $46,7 \%$ des francophones répondent non ; la réponse est « parfois » (« sometimes ») pour 64,9\% des anglophones et 53,3\% des francophones.

« Est-ce que tes parents ou tuteurs t'aident à faire ton travail scolaire ou tes devoirs? ( ( Do your parent(s)/guardian(s) help you with your schoolwork/assignments? »). Cette question aussi a été posée. Cette fois, cependant, francophones et anglophones répondent pareillement ${ }^{36}$. Environ $20 \%$ d'entre eux répondent de façon négative ; plus de $55 \%$ répondent «parfois » (« sometimes »); les autres répondent affirmativement.

«Tes enseignants te donnent-ils de l'aide supplémentaire quand tu en as besoin?» («Do your teachers give you extra help if you need it? »). La répartition des réponses est semblable pour les francophones et les anglophones ${ }^{37}$ : « oui » est majoritaire pour les deux échantillons, avec $67,6 \%$ pour les anglophones et $51,7 \%$ pour les francophones ; « non » est marginal, ne correspondant qu'à $4 \%$ des anglophones et $6,9 \%$ des francophones ; « parfois » apparait chez 28,9\% des anglophones et 41,4\% des francophones.

36. $\chi_{(2)}^{2}=0,63 ; \mathrm{p}=0,73$.

37. $\chi_{(2)}^{2}=2,82 ; \mathrm{p}=0,25$. 
«Est-ce qu'une autre personne t'aide à faire tes devoirs?» ("Does anyone else help you with your schoolwork/assignments? »). Cette autre question a été posée aux élèves. Elle n'a pas permis de distinguer les francophones des anglophones ${ }^{38}$. La réponse est « parfois » (« sometimes ») pour 46,2\% des anglophones et $45,8 \%$ des francophones ; il y a un peu plus d'anglophones qui répondent par l'affirmative $(25,1 \%$ pour $12,5 \%)$ et un peu plus, donc, de francophones qui répondent négativement $(41,7 \%$ pour $28,7 \%)$.

Ainsi, les francophones tendent moins à déclarer nettement qu'ils ont besoin d'aide ou à solliciter cette aide de quelqu'un d'autre que leurs parents ou enseignants, mais ils recourent aussi souvent - quoique de façon sporadique - que les anglophones à l'aide de leurs parents ou de quelqu'un d'autre.

\subsection{Est-on heureux à l'école ?}

Quand on demande aux élèves s'ils sont heureux à l'école, ils répondent majoritairement par l'affirmative, mais les anglophones sont un peu plus nombreux à le faire que les francophones, différence qu'on ne peut attribuer au hasard en fonction des données disponibles ${ }^{39}$. Si les élèves sont inscrits dans les écoles de langue anglaise, ils sont $82,5 \%$ à dire oui ; s'ils sont dans les écoles françaises, leur réponse est semblable dans $63 \%$ des cas. Ce qui distingue les deux échantillons, ce ne sont pas les réponses négatives, qui sont à peu près semblables (14\% pour les anglophones et $11,1 \%$ pour les francophones), c'est le fait que les francophones $(25,9 \%)$ sont plus enclins que leurs camarades de l'autre langue $(3,5 \%)$ à livrer une réponse nuancée : à la fois oui et non.

Si les jeunes répondent non, c'est que l'école leur apparaît monotone, que leurs choix leur semblent limités, que la vie sociale est difficile ou qu'ils éprouvent des difficultés avec les enseignants. S'ils répondent oui, c'est, au premier chef, à cause de la vie sociale - c'est, de loin, la fréquence la plus élevée - ; mais

38. $\chi_{(2)}^{2}=2,61 ; \mathrm{p}=0,27$.

39. $\chi_{(2)}^{2}=19,10 ; \mathrm{p}<0,001$. 
ce peut être aussi parce qu'ils aiment l'école (« enjoy school»), qu'ils s'entendent bien avec leurs enseignants, que leurs cours leur plaisent et qu'ils obtiennent de bonnes notes, qu'ils apprécient l'environnement...

Si l'on demande aux élèves «Qu'est-ce qui te rend heureuse ou heureux à l'école? » ("What makes you happy at school?»), de loin, la réponse la plus commune est celle qui renvoie aux amis ; elle compte pour $76,7 \%$ des réponses exprimées ; elle est dominante chez les francophones comme chez les anglophones. Les autres motifs n'ont pas de fréquence supérieure à $10 \%$; il est question d'activités parascolaires, de notes, de devoirs... Si l'interrogation porte maintenant sur les motifs du malheur, la réponse la plus commune, c'est le travail scolaire ou les devoirs $(27,8 \%)$; en concordance avec les causes du bonheur qui est vécu à l'école, les autres facteurs les plus importants qui rendent malheureux sont le taxage $(19,4 \%)$ et la vie sociale $(9,7 \%)$; les notes et les relations avec les enseignants ou avec le personnel de l'école sont aussi signalées.

Dans le questionnaire, l'élève trouve la question suivante : «Quel est l'objectif principal que tu t'es fixé par rapport à l'école cette année? » («What is your main goal in attending school this year? »). Pour la plupart des élèves, francophones et anglophones, cet objectif a trait à la scolarisation en tant que telle : aux notes ( grades »), aux réalisations («doing well»), au développement («furthering education »). Les autres aspirations sont plus individualisables : les activités parascolaires, obtenir un prix, la vie sociale. Elles sont, certes, moins récurrentes, mais elles ne sont pas pour autant anodines dans l'esprit du jeune. Lisons ce passage d'une entrevue où l'élève s'exprime, entre autres choses, sur les activités parascolaires qui l'attendent :

Élève ${ }^{40}$ :

Oui. Les mathématiques étaient fortes ; le français, l'anglais ; alors ça joue un rôle vraiment important parce que le but de l'école, c'est sûr, est de se faire des amis ; mais le plus haut, c'est l'éducation pour

40. Élève \$1YC2FF020A. 
que tu puisses avoir des connaissances qui vont durer le long de ta vie ; alors l'éducation, ça joue un rôle important ; puis les activités parascolaires. Si deux écoles ont... par exemple, l'éducation est du même niveau, mais qu'une a plus d'activités parascolaires, bien, c'est sûr qu'on va choisir cette école. Parce que les activités parascolaires, par exemple les clubs et tout ça, c'est quelque chose qui n'a pas rapport avec les études; alors, c'est juste un autre monde où tu apprends; mais ce n'est pas en rapport avec les maths, et tout ça. Alors les activités parascolaires, bien c'est important pour moi. Alors les sports... comme les sports sont vraiment amusants et on fait de l'exercice et on apprend la coopération avec les autres personnes ; puis on apprend des éléments clés tels que la coopération, la participation, parce que, des fois, dans les classes, on est un peu timide et on ne veut pas demander une question pour que tout le monde ne nous dise pas, par exemple, que c'est une question stupide ; mais quand tu vas dans un autre club, tu vas voir que, par exemple, une personne qui est vraiment timide va venir et va donner de grandes idées et va participer et va apporter des matériels pour faire tout ça. Alors les activités parascolaires sont vraiment importantes pour moi.

L'école est un lieu d'apprentissage. Les jeunes le savent; les objectifs qu'ils se donnent sont le témoignage de cette vision. Mais l'école n'est pas qu'un endroit où l'on étudie ; c'est aussi un milieu de vie, et les relations avec les autres y occupent un grand espace dans l'esprit des élèves. Les autres, ce sont avant tout les amis, mais ce sont aussi les enseignants et, plus généralement, tout le personnel de l'école. Le plaisir d'aller à l'école, et d'y être, est forcément lié à l'ingestion de quelque mixture faite d'ingrédients scientifiques, artistiques et sportifs dilués dans une forte dose d'amitiés et d'échanges avec des adultes hautement symboliques. 


\subsection{Les notes}

Les élèves qui ont participé à la recherche ont pour moyenne, « jusqu'à présent à l'école » (« at school so far this year »), 80 sur $100^{41}$, avec un écart type d'un peu plus de 8 . Il y a, certes, des variations entre les individus; il n'en demeure pas moins que cette moyenne est élevée. Cela s'explique à nouveau par les contraintes déontologiques auxquelles a dû se plier la recherche, contraintes qui ont sans doute exclu des élèves peu studieux.

\subsection{Choix de l'école secondaire}

Une série de questions porte sur le choix d'une école secondaire. Pour des raisons de logistique, très peu de francophones y ont répondu ${ }^{42}$. Quand les données l'ont permis, nous avons comparé les réponses des francophones à celles des anglophones; nous n'avons alors pas trouvé de différence significative entre les deux échantillons. Puisque, donc, les francophones sont en nombre trop petit pour qu'on effectue continûment des comparaisons et que les comparaisons qui ont été effectuées quand cela était possible ne dévoilent pas de différences, nous avons choisi de réunir les réponses des deux groupes.

$\mathrm{Au}$ moment où ils ont répondu au questionnaire, $93 \%$ des jeunes avaient arrêté leur choix sur une école secondaire.

Nous leur avons demandé d'énumérer les trois principales raisons pour lesquelles ils avaient choisi tel établissement. Chaque élève a donc pu fournir trois raisons. Si l'on additionne les fréquences pour ces trois ensembles de réponses, on peut les hiérarchiser globalement (voir le Tableau 9). Le premier motif (91) a trait à la vie sociale. Viennent en deuxième, par ordre de fréquence à nouveau (74), l'emplacement de l'établissement ou le lien que cette institution entretient avec l'école d'où l'on provient et la famille. Se manifeste ensuite l'offre de cours (71). On notera

41. 3,45 selon l'autre système de notation.

42. Notamment parce que les discussions de groupe en français duraient longtemps. Or, les étudiants répondaient au questionnaire au terme de cette discussion. Comme le temps qui était réservé à toute la séance était limité, il est arrivé que des francophones n'aient pas le temps de répondre aux dernières questions. 
dans ces évocations prioritaires l'immense place qu'occupent dans la conscience des élèves les considérations sociales, familiales et pratiques à côté de préoccupations de type scolaire. En deçà de ces questionnements se révèlent d'autres éléments de réflexion et de décision : les activités parascolaires qui sont envisagées, la réputation de l'école qui accueillera, le fait que cette école soit impressionnante par sa taille ou ses qualités, les aspirations du jeune. Cette énumération montre bien que les facteurs les plus déterminants sont fortement liés aux amitiés, à la famille, au lieu d'habitation et à la structure scolaire.

Si l'on pousse un peu l'interrogation et qu'on demande aux jeunes s'il y a d'autres raisons (« other reasons as well») pour lesquelles ils ont choisi leur école, les pensées scolaires en tant que telles se font plus fréquentes, mais il s'agit d'un second degré de réflexion ou de motivation.

Lisons ce passage d'un entretien où l'élève témoigne du caractère pratico-existentiel de son choix :

Animatrice : $\quad[\ldots]$ Pendant le groupe de discussion toujours, on a parlé de ce qui influençait votre décision d'aller à telle ou à telle école secondaire. Et puis, toi, tu avais indiqué la langue, les amis, les parents et puis ce que l'école a à offrir. [...] Est-ce que tu peux me parler un peu de ça?

Élève ${ }^{43}$ : Bien, la langue, parce que je veux vraiment apprendre en français. Parce que je ne veux pas perdre mon français parce que, moi, c'est important. Puis mes amis, je ne voudrais pas arriver à une école où je ne connais personne. Puis mes parents parce que, bien, mes parents, ils aiment ça que je sois près de l'école. Puis, c'est... puis le... ce que l'école a à offrir, il y a beaucoup... [Petit rire.] Il y a beaucoup d'activités... comme parascolaires ; j'aime faire des activités comme les sports, puis tout ça. Puis il y a aussi le PPCS, le bac international; donc je veux aller dans ça...

43. Élève \$2YC2FF079C. 
Tableau 9

Fréquence des raisons à partir desquelles les élèves justifient le choix de leur établissement pour les études secondaires selon l'ordre du choix

\begin{tabular}{|c|c|c|c|c|}
\hline Raisons & Ordre du c & oix & & Total \\
\hline Type de motif & 1 ère raison & $2^{\mathrm{e}}$ raison & $3^{\mathrm{e}}$ raison & \\
\hline Vie sociale (social) & 31 & 42 & 18 & 91 \\
\hline $\begin{array}{l}\text { Lieu/structure } \\
\text { (location/receiver high school) }\end{array}$ & 41 & 18 & 15 & 74 \\
\hline Famille (family) & 26 & 25 & 23 & 74 \\
\hline L'offre de cours (course selection) & 38 & 17 & 16 & 71 \\
\hline Activités parascolaires (extracurricular) & 10 & 11 & 11 & 32 \\
\hline Réputation (reputation) & 4 & 12 & 13 & 29 \\
\hline Plus grand ou mieux (bigger/better) & 5 & 15 & 9 & 29 \\
\hline $\begin{array}{l}\text { Développement/objectifs } \\
\text { (furthering education/future goals) }\end{array}$ & 6 & 11 & 7 & 24 \\
\hline Pas de choix (no choice) & 16 & 2 & 3 & 21 \\
\hline Nouveau départ (fresh start) & 2 & 2 & 4 & 8 \\
\hline Autre & 3 & 5 & 9 & 17 \\
\hline Total & 182 & 160 & 128 & 470 \\
\hline
\end{tabular}

Lisons aussi ce passage d'une discussion entre des parents et l'animatrice, discussion qui rappelle, entre autres choses, que l'école est une institution dans une communauté, et que ce lien qu'elle entretient avec son milieu est plus important que la variété des choix d'établissement, et qui, de ce fait, ramène au caractère pratique et social du choix d'un établissement :

Animatrice : Est-ce que vous pensez que les amis vont jouer le même rôle quand votre enfant va être au secondaire?

Parent $1^{44}$ : Ce sont les mêmes choses qui vont se produire à ton jeune et vont se produire à ses amis. Ce sont les mêmes stress qui vont les affecter. Alors, dans le fond, la relation reste pareille.

Animatrice: Ce n'est pas le fait d'aller au secondaire qui...

Parent 1: $\quad$ Non. C'est le fait de vieillir, de grandir.

[Acquiescement.]

44. Parent \$6PL1FF003A. 
Parent 1: $\quad$ Là, peut-être qu'il y a quelque chose ; c'est juste pour qualifier : c'est que nous, à [nom de ville], vu la façon que l'éducation... comme je veux dire : on est chanceux, les écoles sont toutes proches. Ce n'est pas comme s'il fallait changer de quartier pour aller à l'école ou changer de groupe social ou ethnique, ou rien de ça. Ce sont les mêmes jeunes qui transfèrent d'une école à l'autre. Ça, c'est un gros plus.

[Acquiescement.]

Animatrice : C'est en partie pourquoi il n'y a pas de... nécessairement de transformations au niveau de l'influence des amis parce que...

[Acquiescement.]

Animatrice : Les amis restent les mêmes; ils sont à l'élémentaire avec...

Parent $2^{45}$ : $\quad$ Ils se suivent depuis la maternelle, cette gang-là.

Parent 1: Oui. Ils sont dans les mêmes classes, les mêmes groupes sociaux. Et, l'autre affaire, bien, ça peut être sur un côté ou l'autre, mais il n'y a pas de compétition d'école, ici. Il n'y a qu'une école secondaire ; malgré les deux ou trois conseils qui sont représentés, il n'y a qu'une école secondaire. Cela est déjà une chose qui fait un stress de moins.

Animatrice: Ah oui ! Vous voyez cela comme un stress de moins?

Parent 1: $\quad$ Ah oui ! Définitivement.

Animatrice : C'est ce que j'allais vous demander, tout à l'heure, justement...

Parent 2: Oui, mis comme ça, il n'y a probablement qu'une question que j'aurais cochée : « qui n'avait pas d'importance », mais, oui, finalement, cela avait de l'importance...

Parent 1: $\quad$ C'est tout personnel...

Parent 2: Oui.

Parent 1: C'est de la façon que... on le voit.

Parent 2: Oui, c'est ça. Tu viens de mentionner ça, et ça fait du sens.

45. Parent \$6PL2FF002A. 
Parent 1: J'imagine mon jeune... pas loin, [nom de ville], [nom de ville]; tu as le choix entre deux écoles, trois écoles; et, que ce soit le conseil que ça voudra, tu as le choix de trois écoles, mais ça peut vraiment vouloir dire une différence dans ta scolarité, où tu vas aller dans la vie. [Nom de ville], il y a quatre, cinq, six écoles secondaires? Ah oui ! il y en a plusieurs. Alors, ce qui arrive, c'est que ça peut faire des stress de plus.

Animatrice : Mais, ça, c'est intéressant qu'ils ont mis ça comme un stress de moins, parce que, justement, une des questions que j'allais vous poser, c'est comme... justement, quand on va à [nom de ville], on voit qu'il y a des élèves qui ont un choix entre une dizaine d'écoles et, dans certains cas, ça veut dire un transport en commun qu'ils n'avaient pas avant ; ça veut dire des fois une heure, quarantecinq minutes. Ça veut dire un changement d'amis ; ça veut dire toutes sortes de choses ; et puis, eux le formulent à la fois comme un stress et comme quelque chose d'excitant; et un des termes qui revient souvent, c'est « nouveau départ »-ils le vivent un peu comme ça; c'est une chance de me faire des nouveaux amis et de repartir. C'est vrai que, quelque part, on s'imagine l'enfant qui ne se sent pas intégré, qui n'est pas bien ou qui n'aime pas son école élémentaire pour quelque raison que ce soit ; pour eux, c'est une chance de renouveau; alors je me suis posé la question : ici, c'est un peu particulier, quand je viens ici, et à [nom de ville], j'ai un groupe de discussion et je me demande si les parents ont peut-être, eux, l'impression que le fait qu'il y ait juste une école secondaire est une lacune. Je me demande si les parents aimeraient que les élèves aient un choix. Mais, d'après ce que vous me dites, il y a certainement un côté très positif à ça, que, vous, vous voyez en bien; ils se suivent, les amis restent les mêmes, ou à peu près.

Parent 1: Je pense que ce qui arrive... encore là, on a fait un choix personnel de demeurer ici pour ces 
raisons-là. Alors ce n'est qu'une continuation de notre choix personnel où on choisit de vivre ou de travailler.

Animatrice: C'est bien dit, oui.

Parent 1: On ne voit pas ça comme une restriction. On ne voit pas ça non plus comme une grosse opportunité. On voit ça comme une zone de confort.

Animatrice : Ça participe de votre réflexion générale que vous... Parent $3^{46}$ : $\quad$ Oui, parce que, nous autres mêmes, on est parti d'ici et on est allé...

Parent 1: $\quad$ On est allé essayer à l'extérieur.

Parent 2: Oui, bien, moi, je suis allé au collège à l'extérieur ; nous-mêmes avons fait notre huitième ici et on est allé au secondaire... si j'aurais aimé aller ailleurs? je ne pense pas. Avoir eu le choix entre plusieurs écoles? Je ne pense pas.

Parent 1: On suit nos amis, notre culture. On suit notre groupe.

Parent 2: $\quad$ Ça fait un gros stress de moins. Pour certains jeunes, ils sont capables de le prendre; pour d'autres, non.

\subsection{Sentiments attachés à la transition}

Durant les discussions de groupe, les jeunes ont été appelés à faire état de leurs sentiments à l'égard de la transition. À un moment donné, si le temps le permettait, les animatrices leur présentaient une feuille sur laquelle se trouvent des visages exprimant des émotions. Les jeunes devaient alors encercler des visages, autant qu'ils le voulaient, qui caractérisent leur état d'âme. En moyenne, ils en ont encerclé $3,89(\mathrm{~s}=1,84)$; cette statistique révèle qu'on n'a pas affaire à une problématique qui soit facilement réductible au plan affectif.

Six émotions semblent, plus que les autres, traduire le vécu des élèves (voir le Tableau 10). D'abord le bonheur puis l'espoir ; la perspective des études secondaires rend heureux et génère des visions optimistes. Ensuite, l'inquiétude et la peur ; le bonheur et

46. Parent \$6PL2FF001A. 
l'optimisme cohabitent souvent avec la crainte, une crainte largement alimentée par l'inconnu. Puis l'assurance et la confusion ; si l'on est à certains égards sûr de soi, ce n'est pas parce que l'esprit n'est que certitude. Ces six émotions sont dominantes; au sein d'un même esprit, elles cohabitent, communément en entretenant des tensions paradoxales. Entrevoir les études secondaires, c'est certainement éprouver des sentiments au pluriel ; mais, chez les jeunes qui se sont livrés à nous, la gêne, la colère, la rage ou le dégoût n'en font pas partie. Peuvent s'ajouter toutefois aux sentiments dominants la tristesse, l'accablement, la prudence, la méfiance, sous diverses formes.

Quelques aspirations, quelques émotions exprimées par des élèves :

Élève $1^{47}$ : Le plein d'espoir ! J'ai plein d'espoir parce que je veux beaucoup accomplir au secondaire parce que ce sont des années dont tout le monde dit que ce sont les meilleures années de notre vie, avec celles de l'université. Alors, je veux vraiment que le secondaire ne soit pas comme : « ah ! c'est plate; on a beaucoup de devoirs », mais que ça soit vraiment amusant et qu'il y ait, genre, une balance d'activités et d'études. Je veux accomplir beaucoup au cours de ces années.

$$
* * *
$$

Élève 1 : $\quad[\ldots]$ quand on est enfant, on n'a pas beaucoup de responsabilités; et puis on est plus libre. On ne pense pas au futur, et tout ça ; on s'amuse à jouer au basketball, et tout ça ; mais, là, quand tu viens en huitième année, il y a les professeurs qui viennent te parler des crédits et puis, disons, des projets à faire ; alors on se sent triste ; bien, moi, je me sens triste parce que je ne veux pas quitter mon enfance. Je veux toujours aller prendre de la

47. Élève $\$ 1 Y C 2 F F 020 A$. 
crème glacée avec mon père, et jouer. Je me sens comme si je vais au secondaire, ça va tout changer et je vais être considérée comme une adulte et être parlée comme une adulte ; et je ne veux pas ça ; je veux toujours être une enfant. C'est ça, alors, c'est dans ces années qu'on mature, et on ne quitte pas notre enfance ; mais, je ne sais pas comment l'expliquer, mais je me sens triste parce que je veux être encore l'enfant de cinq ans ; mais je ne peux pas retourner. Alors, maintenant, je suis en face de mon futur et je ne veux pas quitter l'enfance comme...

Animatrice : Est-ce que tu penses que tu as à la quitter?

\section{Tableau 10}

Encerclement ou non de visages exprimant des émotions en tant qu'elles caractérisent la manière dont se sent l'élève à l'égard de la transition vers le secondaire

\begin{tabular}{|c|c|c|c|}
\hline \multicolumn{4}{|l|}{$\begin{array}{l}\text { (Réponse en centièmes) } \\
n=37\end{array}$} \\
\hline \multirow[t]{2}{*}{ Expression du visage } & \multicolumn{2}{|c|}{ Visage encerclé } & \multirow[t]{2}{*}{ Total } \\
\hline & oui & non & \\
\hline Heureux (happy) & 0,60 & 0,40 & 1,00 \\
\hline Sûr de soi (confident) & 0,35 & 0,65 & 1,00 \\
\hline Plein d'espoir (hopeful) & 0,46 & 0,54 & 1,00 \\
\hline Fou de joie (ecstatic) & 0,14 & 0,86 & 1,00 \\
\hline Méfiant (suspicious) & 0,14 & 0,86 & 1,00 \\
\hline Prudent (cautious) & 0,19 & 0,81 & 1,00 \\
\hline Ennuyé (bored) & 0,08 & 0,92 & 1,00 \\
\hline Confus (confused) & 0,30 & 0,70 & 1,00 \\
\hline Inquiet (anxious) & 0,43 & 0,56 & 1,00 \\
\hline Accablé (overwhelmed) & 0,22 & 0,78 & 1,00 \\
\hline Hystérique (histerical) & 0,05 & 0,95 & 1,00 \\
\hline Dégoûté (disgusted) & 0,00 & 1,00 & 1,00 \\
\hline Apeuré (frightened) & 0,41 & 0,59 & 1,00 \\
\hline Fâché (angry) & 0,00 & 1,00 & 1,00 \\
\hline Enragé (enraged) & 0,00 & 1,00 & 1,00 \\
\hline Frustré (frustraged) & 0,05 & 0,95 & 1,00 \\
\hline Déprimé (depressed) & 0,03 & 0,97 & 1,00 \\
\hline Triste $(\mathrm{sad})$ & 0,19 & 0,81 & 1,00 \\
\hline Seul (lonely) & 0,14 & 0,86 & 1,00 \\
\hline Gêné (embarassed) & 0,00 & 1,00 & 1,00 \\
\hline
\end{tabular}


Élève 1: $\quad$ Bien [hésitation], c'est qu'on ne va pas la quitter ; comme, moi, je me sens comme si... ce n'est pas que tu vas la quitter; tu as quand même treize ans ; tu es quand même considéré comme un mineur, comme un enfant ; c'est juste que les décisions qu'on prend, ça doit être plus sérieux et on ne veut pas toujours être sérieux ; alors, d'un côté, on quitte l'enfance un peu : on mature et on devient plus responsable, mais, de l'autre côté, on peut quand même s'amuser, et tout ça. C'est juste comme un équilibre des deux.

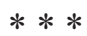

Animatrice : $\quad[\ldots]$ Quand on a parlé de ce qui serait le plus difficile, dans le fait d'aller au secondaire, tu avais indiqué : beaucoup de pression. Est-ce que tu peux me parler des pressions?

Élève $2^{48}$ : Niveau de pression sur nous parce qu'on veut graduer de la douzième année; alors : «Travaille bien », «Tu as tout fait tes travaux? », « Dépends sur toi », alors : « Travaille bien », tout ça. Alors, là, ça commence à te mettre un peu plus de pression ; puis, chaque année, ils vont te dire la même chose : «Travaille bien, travaille bien, ton futur dépend de toi »; et puis, là, c'est vraiment la personne qui va y penser : " oui, mon futur est entre mes mains ; alors si je ne travaille pas bien, tu n'auras pas de futur, ou un bon futur ». C'est bon de mettre un peu de pression, mais pas beaucoup. Je ne sais pas s'ils vont mettre d'autre pression, mais j'attends qu'ils, mes parents, vont commencer à mettre de la pression, et crois-moi... Un peu, pas trop, mais ils vont commencer, je pense, dès l'année prochaine : ils vont dire « Heille! travaille bien; étudie ; fais ça, fais ci, montre-moi ça ». Ils vont commencer à vouloir... à chaque jour, ils veulent voir mes devoirs, ils veulent voir si je fais bien.

48. Élève \$1YC2FF021A. 


\section{Conclusion}

La meilleure façon de comprendre un groupe social, c'est de repérer ce qui le rend semblable aux autres et ce qui l'en distingue. La meilleure façon d'intervenir efficacement auprès d'une population particulière, c'est avant tout d'en saisir les particularités ; et donc de découvrir en quoi elle est ou non assimilable aux autres. Et cette information est d'autant plus importante que cette population est minoritaire. Car il est deux façons de servir un groupe minoritaire de façon adéquate : lui fournir les services dont il a besoin en fonction de sa spécificité ; lui fournir les services auxquels a droit la majorité. Dans cet esprit, nous avons choisi de décrire le phénomène du passage des jeunes francophones de l'élémentaire au secondaire en le comparant à celui de leurs camarades anglophones.

La question principale à laquelle nos analyses ont voulu apporter quelque réponse est celle de la comparabilité des élèves francophones ou anglophones avant la transition vers l'école secondaire (ou le changement d'école) et en fonction de cette transition.

Nous avons réparti les observations en 14 sections.

i. La première dessine le profil des échantillons. Elle indique que : sur le plan ethnique, l'ensemble francophone est moins bigarré que l'ensemble anglophone ; les élèves sont relativement heureux, qu'ils soient anglophones ou francophones ; la plupart des élèves n'ont pas l'impression de faire partie d'un groupe qui souffre de discrimination ; 1'échantillon francophone est plus bilingue que l'anglophone ; les parents des élèves des deux échantillons sont plus instruits que ne le sont les adultes dans les populations dont ils sont issus.

ii. La deuxième révèle que les amis occupent un espace important dans l'imaginaire des élèves, indépendamment de leur langue.

iii. La troisième dénote que plusieurs jeunes trouvent qu'ils ont l'air plus grand, ou plus petit, ou plus vieux que leurs 
collègues ; un nombre important de ces jeunes s'estiment plus responsables que leurs camarades de classe. Cela vaut pour les francophones et les anglophones.

iv. La quatrième relève que les francophones et les anglophones éprouvent de façon comparable leur environnement scolaire, les uns ne se sentant pas vraiment plus étrangers que les autres dans leur milieu, ne s'y sentant pas vraiment plus à leur aise, n'ayant pas moins l'impression de faire partie du groupe.

v. La cinquième section indique que francophones et anglophones posent un regard semblable sur leur école, un regard qui est satisfait, mais qui n'est pas comblé.

vi. La sixième section révèle que les jeunes estiment que la population étudiante de leur école est quelque peu diversifiée et qu'elle l'est davantage que la population enseignante. Elle ne relève pas de différence entre francophones et anglophones.

vii. La septième section montre que les élèves considèrent que leurs enseignants sont plutôt enclins à les appuyer dans leur apprentissage, à rendre l'apprentissage amusant, à se soucier d'eux, à faire attention à leurs amis, à se rendre accessibles, à leur fournir des renseignements sur 1'école secondaire, à les aider à prendre des décisions à l'égard de l'école secondaire ; mais les positions ne sont pas unanimes. Il n'y a pas de différence entre francophones et anglophones.

viii. La huitième section signale que les jeunes, de leur propre aveu, sont entourés de parents et d'enseignants qui les encouragent à bien travailler. Francophones et anglophones pensent pareillement.

ix. La neuvième dénote que les jeunes se représentent de façon hautement positive les études secondaires et que, selon eux, il en va de même de leurs parents. Cette image est un peu moins nette pour leurs amis. 
x. La dixième section indique que les francophones tendent moins que les anglophones à déclarer clairement qu'ils ont besoin d'aide ou à solliciter cette aide de quelqu'un d'autre que leurs parents ou leurs enseignants.

xi. La onzième section met en évidence le fait que, pour les élèves, l'école est aussi bien un milieu de vie qu'un lieu d'apprentissage et que le plaisir d'y être est fortement tributaire des relations qu'ils entretiennent avec leurs amis et tout le personnel de l'école. Cela vaut pour les deux groupes linguistiques.

xii. La douzième indique que les notes moyennes des élèves qui ont participé à l'étude sont très élevées.

xiii. La treizième section révèle que l'immense majorité des élèves avaient choisi leur école secondaire au moment de la collecte de données. Elle montre que les motifs qui président à ce choix sont normalement d'ordre amical, pratique et familial.

xiv. La quatorzième section, enfin, indique que les sentiments que les jeunes éprouvent en songeant aux études secondaires sont généralement nombreux et souvent contradictoires : le plaisir et l'espoir cohabitent communément avec la peur et l'inquiétude.

On sait que des jeunes franco-ontariens se déplacent des écoles de langue française vers les écoles de langue anglaise et que ce transfert a lieu principalement au moment où une transition devient nécessaire, soit généralement quand ils passent de l'élémentaire au secondaire, mais aussi lorsque la sixième année est terminale dans un établissement spécifique. On sait aussi que ce mouvement, sans être majoritaire, est important, suffisamment, en tout cas, pour alerter les leaders et les chercheurs de l'Ontario français.

Les premières études sur les représentations des élèves francophones qui sont sur le point de vivre une transition montrent que se 
côtoient une idéologie pro-française et une autre, pro-anglaise. Le décrochage culturel s'alimente à cette seconde vision du monde. Des travaux ultérieurs devront étudier en profondeur cette dualité des discours aussi bien dans ses contenus qu'en fonction de ses modes de reproduction et de développement.

La coexistence de ces idéologies est suffisamment active dans la communauté francophone de l'Ontario pour orienter concrètement deux types de comportements. Si elle est active dans la communauté anglophone, ce ne peut être avec la même intensité ou la même étendue. Dans les faits, on n'en trouve pas de présence significative quand on examine le discours des jeunes anglophones ${ }^{49}$.

Quand ils sont placés devant l'obligation de la transition, les jeunes francophones diffèrent-ils des anglophones sur d'autres points ? La réponse tend à être négative. Le système d'éducation uniformise à bien des égards les uns et les autres. Pour les deux ensembles, les considérations affectives et pratiques sont hautement décisives : les amis sont importants, la proximité familiale joue pour beaucoup, l'éloignement du domicile entre en jeu. On ne peut pas affirmer que la langue maternelle soit réellement déterminante du bonheur ou du malheur, que les parents de tel échantillon semblent meilleurs que ceux de tel autre. On ne peut pas déclarer non plus que les jeunes qui étudient dans telle langue apprécient davantage leur école. On a affaire à une forte homogénéité sur ces plans. Il ne nous semble pas par ailleurs que nous aurions trouvé plus de différences entre les deux groupes linguistiques si nos échantillons avaient compté plus d'élèves qui proviennent des milieux les moins bien nantis : la pauvreté des francophones n'est pas à ce point éloignée de la pauvreté des anglophones que les jeunes qui en sont issus auraient des représentations sociales et scolaires, des vécus familiaux à l'opposé de leurs camarades anglophones.

49. Kate Tilleczek, Simon Laflamme, Bruce Ferguson, Dara Roth Edney, Mélanie Girard, Dana Cudney, Siobhan Cardoso, op. cit. 
Il faut donc conclure que la dualité idéologique trouve son origine ailleurs, que la particularité du discours francophone se construit sur d'autres bases. Il nous semble que, pour en saisir la formation, il faudrait se pencher sur chacune de ses expressions et trouver qui en sont les promoteurs et quels sont les réseaux à l'intérieur desquels elles circulent. On pourrait, par exemple, interroger les jeunes sur les termes des idéologies et interroger pareillement leurs amis, parents et enseignants, puis aménager un ensemble de variables qui, théoriquement, devraient permettre de dessiner des profils associables aux diverses manifestations discursives. Une idéologie ne peut se développer qu'à l'intérieur de relations ; une idéologie ne peut se construire que dans un ensemble d'informations qui lui donnent sa forme. Ces relations sont sociales ; ces informations sont sociales. L'idéologie est le résultat d'interactions entre des personnes et d'exposition à des messages, médiatisés ou non. L'enquête qui voudrait comprendre le développement de l'idéologie duelle franco-ontarienne devrait avoir ces principes à l'esprit; elle devrait donc tenter de découvrir un rapport entre des personnes et un discours, entre des catégories de personnes, entre des types de contenus informatifs; elle devrait, en outre, trouver les sources de ces discours et établir les caractéristiques de ces personnes. 